Draft Version November 20, 2018

Preprint typeset using $\mathrm{LAT}_{\mathrm{E} X} \mathrm{X}$ style emulateapj v. 03/07/07

\title{
THE SPATIAL AND VELOCITY DISTRIBUTIONS OF HYPERVELOCITY STARS
}

\author{
Fupeng Zhang ${ }^{1}$, Youjun Lu ${ }^{1}$, AND QinguUAN Yu ${ }^{2}$ \\ ${ }^{1}$ National Astronomical Observatories, Chinese Academy of Sciences, Beijing, 100012, China; luyj@nao.cas.cn \\ ${ }^{2}$ Kavli Institute for Astronomy and Astrophysics, Peking University, Beijing, 100871, China; yuqj@kiaa.pku.edu.cn
} Draft version November 20, 2018

\begin{abstract}
Hypervelocity stars (HVSs) found in the Galactic halo are believed to be the dynamical products of interactions between (binary) stars and the massive black hole(s) (MBH) in the Galactic center (GC). In this paper, we investigate how the spatial and velocity distributions of HVSs are connected with their originations, ejecting mechanisms, and the dynamical environment in the GC. It has been shown that the detected HVSs are spatially consistent with being located on two thin disks (Lu et al.), one of which has the same orientation as the clockwise rotating stellar disk in the GC. Here, we perform a large number of three-body experiments of the interactions between the MBH and binary stars bound to it; and we find that the probability of ejecting HVSs is substantially enhanced by multiple encounters between the MBH and binary stars at a distance substantially larger than their initial tidal breakup radii. Assuming that the HVS progenitors are originated from the two thin disks, our simulations show that the distributions of the HVS inclination relative to the disk planes can be well reproduced by either the mechanism of tidal breakup of binary stars or the mechanism of ejecting HVSs by a hypothetical binary black hole (BBH) in the GC. However, an isotropical origination of HVS progenitors is inconsistent with the observed inclination distribution. Assuming that the detected HVSs were ejected out by tidal breakup of binary stars, its velocity distribution can be reproduced if their progenitors diffuse onto low angular momentum orbits slowly and most of the progenitors were broken up at relatively large distances because of multiple encounters. Assuming that the HVSs were ejected out by a BBH within the allowed parameter space in the GC, our simulations produce relatively flatter spectra at the high-velocity end compared to the observed ones; however, the BBH mechanism cannot be statistically ruled out, yet. Future deep surveys of HVSs and better statistics of the HVS spatial and velocity distributions should enable to distinguish the ejection mechanisms of HVSs and shed new light on the dynamical environment surrounding the central MBH.
\end{abstract}

Subject headings: Black hole physics - Galaxy: center-Galaxy: halo-Galaxy: kinematics and dynamics-Galaxy: structure

\section{INTRODUCTION}

Hypervelocity stars (HVSs) discovered recently are mainly B type stars with mass $\sim 3-4 M_{\odot}$ and radial velocities up to $700 \mathrm{~km} \mathrm{~s}^{-1}$ escaping away from the Galactic halo (Brown et al. 2005; Hirsch et al. 2005; Edelmann et al. 2005; Brown et al. 2007, 2009a; Bromley et al. 2009). These HVSs are believed to be ejected out of the Galactic center (GC) by dynamical interactions of (binary) stars with the central massive black hole(s) (MBH; Brown et al. 2009a, b)). There are mainly three mechanisms that can produce such highvelocity stars: (1) the tidal breakup of binary stars in the immediate vicinity of the central MBH (hereafter TBK mechanism; Hills 1988; Yu \& Tremaine 2003; Bromley et al. 2006; Sari et al. 2010); (2) three-body interactions of single stars with a hypothetical low-mass ratio binary black holes (BBHs) in the GC (hereafter, BBH mechanism; Yu \& Tremaine 2003; Gualandris et al. 2005; Levin 2006; Baumgardt et al. 2006; Sesana et al. 2006; Löckmann \& Baumgardt 2008); and (3) interactions of single stars with a cluster of stellar mass black holes in the vicinity of the central MBH (hereafter, SBHs mechanism; O'Leary \& Loeb 2008). ${ }^{1}$

1 Alternative models, other than the GC origin, are also proposed to explain the HVSs by Heber et al. (2008), Abadi et al. (2009), Tutukov \& Fedorova (2009), and Wang \& Han (2009).
To determine which mechanism should be responsible for the detected HVSs is an important issue in understanding the stellar dynamics around the central MBH. Some observational properties of HVSs, such as the binarity, rotational velocity, metallicity, ejection rate/observed frequency, spatial and velocity distribution, etc., are proposed to be helpful in distinguishing these mechanisms (e.g., Lu et al. 2007; Hansen 2007; Sesana et al. 2007; Przvbilla et al. 2008; López-Morales \& Bonanos 2008; Perets 2009a, b). However, one uncertain factor in these mechanisms is where the initial progenitors come from. Different origins of the HVS progenitors may also result in different distributions of HVS properties, especially their spatial distribution and the velocity distribution. The main aim of this paper is to address this problem through Monte Carlo numerical simulations under the constraints from current observations.

The spatial distribution of the detected HVSs is compatible with both the TBK and the BBH mechanism if the progenitors of these HVSs are originated from disk-like structures in the vicinity of the central $\mathrm{MBH}$ (e.g., the clockwise rotating young stellar (CWS) disk in the GC). The preliminary results that demonstrate the $\operatorname{disk}(\mathrm{s})$ origination of the HVS progenitors have been summarized in Lu et al. (2010). We shall further demonstrate in this paper that an isotropic distribution of HVS 
progenitors is inconsistent with the spatial distribution of the detected HVSs if they are originated from the GC. However, the disk(s) origination of the detected HVSs is consistent with the distribution of the inclination angles (relative to the disk(s)) of the detected HVSs which further strengthen the conclusions made in Lu et al. (2010).

The velocity distribution of HVSs is related to not only the production mechanism but also the origin of their progenitors. Sesana et al. (2007) have studied the velocity distribution of HVSs. They found that the velocity distribution of HVSs produced by the TBK mechanism for unbound injecting stellar binaries seems to be consistent with the then detected HVSs though with limited statistics, while the HVS velocity distribution produced by the BBH mechanism appears to be too flat in comparison with the observations. Their results suggest that the HVS velocity distribution may be useful in distinguishing the ejection mechanisms. In this paper, we shall further investigate the effects on the velocity distribution of HVSs due to different origins of the HVS progenitors, e.g., those (binary) stars initially unbound to the $\mathrm{MBH}$ but later injected into the immediate vicinity of the $\mathrm{MBH}$ due to some unknown perturbations, and those (binary) stars initially bound to the $\mathrm{MBH}$ but later evolved onto highly eccentric orbits and migrated into the immediate vicinity of the $\mathrm{MBH}$.

The paper is organized as follows. In Section 2, we first summarize the observational results on the spatial and velocity distribution of the detected HVSs. In Section 3. we explore the detailed dynamics of interactions between binary stars on bound orbits and a central $\mathrm{MBH}$. The consequences of these interactions are different from that between the unbound binary stars on parabolic (or hyperbolic) orbits and the MBH intensively investigated in the literature (e.g., Hills 1988; Bromley et al. 2006; Sesana et al. 2007). The reason is that the stellar binary may experience multiple close encounters with the $\mathrm{MBH}$ in the former case, while it only experiences a single close encounter in the latter case. Assuming realistic distributions of the properties of the initial stellar binaries, we then simulate both the spatial distribution and the velocity distribution of HVSs produced by the TBK mechanism and compare the numerical results with the observations in Section 4. ${ }^{2}$ In Section [5, we also explore the interactions between single stars on bound orbits with a hypothesized BBH in the GC. These single stars are assumed to be injected into the immediate vicinity of the BBH from disk-like stellar structures (e.g., the CWS disk) surrounding the BBH, which is different from that adopted in Sesana et al. (2007). With reasonable but simple assumptions on the parameters of the hypothetical $\mathrm{BBH}$, the spatial and velocity distributions of the ejected HVSs are obtained. Comparison between the simulation results and the observations are also discussed in Section 5. The conclusions are given in Section 6 .

\section{SPATIAL AND VELOCITY DISTRIBUTIONS OF THE DETECTED HVSS}

Surveys of HVSs have detected 16 HVSs unbound to the Galactic halo, 8 bound HVSs, and 4 HVS candidates (Brown et al. 2005; Hirsch et al. 2005; Edelmann et al.

2 The cases of interactions between unbound binaries and the central MBH have been extensively studied by Bromley et al. (2006).
2005; Brown et al. 2007, 2009a). We summarize their spatial and velocity distributions in this section.

\subsection{Spatial distribution}

The spatial distribution of the HVSs detected so far is probably anisotropic Abadi et al. 2009; Brown et al. 2009b). Lu et al. (2010) use great circles to fit the spatial distribution of the detected HVSs projected on the sky of an observer located at the GC, and they find that the distribution can be best fitted by two great circles. Their results suggest that the spatial distribution of the detected HVSs is consistent with being located on the planes of two thin disks (Lu et al. 2010): (1) eleven of the unbound HVSs (plus four bound ones and two candidates; totally 17 objects) are spatially associated to a thin disk plane with an orientation almost the same as that of CWS disk located within half a parsec from the central MBH (see Levin \& Beloborodov 2003; Lu et al. 2009; Paumard et al. 2006; Bartko et al. 2009, 2010); (2) four of the unbound HVSs (plus three bound ones and two candidates; totally 9 objects) are spatially associated to a thin disk plane with an orientation similar to that of the northern arm of the minispiral (or also the outer warped part of the CWS disk) in the GC. The normals of the best-fit disk planes for these two HVS populations are $(l, b)=\left(311^{\circ},-14^{\circ}\right)$ and $\left(176^{\circ},-53^{\circ}\right)$ in Galactic coordinates, respectively (Lu et al. 2010). Hereafter, we refer to those detected HVSs associated with the above two best-fit planes as the first population and the second population of HVSs, respectively. We denote the inclination angle of each HVS to its corresponding best-fit plane by $\Theta$ and describe the spatial distribution of the HVSs by a normalized cumulative distribution function of their inclination angles $P(\geq \Theta)$ (hereafter, $\Theta \mathrm{CDF})$, which represents the number fraction of the HVSs with inclination angles higher than $\Theta$. The observational $\Theta C D F$ s for both populations of the HVSs are shown in Figure 1 and will be compared with the distributions obtained from numerical models in Sections 4 and 5. For each population, we shall compare the $P(\geq \Theta)$ of all the HVSs (including unbound HVSs, bound HVSs, and HVS candidates) instead of only unbound ones, because (1) for the first HVS population, our Kolmogorov-Smirnov (K-S) test finds a likelihood of 0.94 that the unbound HVSs and all the HVSs are drawn from the same $\Theta C D F$; (2) for the second population, the number of the unbound HVSs is only 4 and the error due to Poisson noise in the $\Theta C D F$ is substantial, therefore we do not show their $\Theta C D F$ in Figure 1 (and $v \mathrm{CDF}$ in Figure 2 below, either).

The gravitational potential of the Galaxy is not exactly spherical, and its non-spherical component may deflect the radial trajectories of HVSs after they were ejected from the GC (e.g., Yu \& Madau 2007). Given the distance and the velocity span $(30 \mathrm{kpc}<\mathrm{R}<130 \mathrm{kpc}$ and $690 \mathrm{~km} \mathrm{~s}^{-1}<\mathrm{v}<980 \mathrm{~km} \mathrm{~s}^{-1}$, see Section 2.2) of the detected HVSs, however, the deviation due to the flattening of the Galactic disk and the triaxiality of the Galactic halo is at most several degrees (typically $2^{\circ}$ ) as demonstrated in Yu \& Madau (2007). Compared with the standard deviation of $\Theta\left(\sim 7^{\circ}-8^{\circ}\right)$ shown in Figure 1. that small deviation can be ignored.

\subsection{Velocity distribution}


The detected HVSs have been decelerated in the Galactic gravitational potential after they were ejected from the GC. To easily compare with results obtained from theoretical models and see the dependence of HVS velocity distributions on different ejection mechanisms, we first remove the velocity deceleration (caused by the Galactic bulge, the disk, and the halo; see below) from their observed values with correction for the proper motion and then obtain their velocities at infinity by assuming that they move from the GC to the infinity only in the gravitational potential of the central $\mathrm{MBH}$. We denote these HVS velocities at infinity by $v_{\mathrm{ej}}^{\infty}$, and their $\mathrm{CDF} P\left(\geq v_{\mathrm{ej}}^{\infty}\right)$ (hereafter, $v \mathrm{CDF}$ ) represents the number fraction of HVSs which have velocities at infinity higher than $v_{\text {ej }}^{\infty}$.

Generally, the Galactic gravitational potential can be described by four components, $\Phi=\Phi_{\mathrm{BH}}+\Phi_{\text {bulge }}+\Phi_{\text {disk }}+$ $\Phi_{\text {halo }}$, i.e., the contribution from the central $\mathrm{MBH}$, the Galactic bulge, the disk, and the halo. We have $\Phi_{\mathrm{BH}}=$ $-G M_{\bullet} / r$, where the mass of the central $\mathrm{MBH}$ in the $\mathrm{GC}$ is adopted to be $M_{\bullet}=4 \times 10^{6} M_{\odot}$ (e.g., Ghez et al. 2008; Gillessen et al. 2009). In this paper, we adopt the model for the last three components from Xue et al. (2008), i.e.,

$$
\Phi_{\text {bulge }}=-\frac{G M_{\text {bulge }}}{r+r_{\text {bulge }}},
$$

with $M_{\text {bulge }}=1.5 \times 10^{10} M_{\odot}$ and the core radius $r_{\text {bulge }}=$ $0.6 \mathrm{kpc}$,

$$
\Phi_{\mathrm{disk}}=-\frac{G M_{\mathrm{disk}}\left(1-e^{-r / b}\right)}{r},
$$

with $M_{\text {disk }}=5 \times 10^{10} M_{\odot}$ and the scale length $b=4 \mathrm{kpc}$, and

$$
\Phi_{\text {halo }}=-\frac{4 \pi G \rho_{\mathrm{s}} r_{\mathrm{vir}}^{3}}{c^{3} r} \ln \left(1+\frac{c r}{r_{\text {vir }}}\right),
$$

with $\rho_{\mathrm{s}}=\frac{\rho_{\mathrm{c}} \Omega_{\mathrm{m}} \Delta_{\mathrm{vir}}}{3} \frac{c^{3}}{\ln (1+c)-c /(1+c)}$, where $\rho_{\mathrm{c}}$ is the cosmic critical density, $\Delta_{\mathrm{vir}}=200, \Omega_{\mathrm{m}}$ is the cosmic fraction of matter, the virial radius $r_{\mathrm{vir}}=267 \mathrm{kpc}$, and the concentration $c=12$. To see the velocity deceleration of a star caused by the bulge, disk and halo potentials above, the minimum velocity required for the star starting from the potential center (in the absence of the $\mathrm{MBH}$ ) is $\sim 645 \mathrm{~km} \mathrm{~s}^{-1}$ (or $670 \mathrm{~km} \mathrm{~s}^{-1}, 700 \mathrm{~km} \mathrm{~s}^{-1}$ ) so that they can move to a distance of $50 \mathrm{kpc}$ (or $100 \mathrm{kpc}$, $200 \mathrm{kpc}$ ) further away. With the Galactic potential model above, we correct the velocity deceleration and obtain the $v$ CDFs for both HVS populations, which are shown in Figure 2.

In the estimation of $v_{\mathrm{ej}}^{\infty}$ above, we adopted the spherical approximation of the Galactic disk potential given by Equation (2), since all the detected HVSs are moving at Galactocentric distances much larger than the disk scale length $b$. If using the Miyamoto-Nagai potential (Mivamoto \& Nagai 1975) to describe the flattened potential of the Galactic disk, i.e., $\Phi(R, z)=$ $-G M_{\text {disk }} /\left(R^{2}+\left(a_{\text {disk }}+\left(z^{2}+b_{\text {disk }}^{2}\right)^{1 / 2}\right)^{2}\right)^{1 / 2}$ and $M_{\text {disk }}=$ $5 \times 10^{9} M_{\odot}$, the scale length and height of the disk are $a_{\text {disk }}=5 \mathrm{kpc}, b_{\text {disk }}=0.4 \mathrm{kpc}$, respectively, we find that the difference in the estimated $v_{\mathrm{ej}}^{\infty}$ due to the disk flattening is negligible $\left(\sim 10 \mathrm{~km} \mathrm{~s}^{-1}\right)$.

Adopting a different Galactic potential model may result in a different $v$ CDF. For example, choosing the Galactic potential as that given by Equation (8) in Kenvon et al. (2008), the resulted $v_{\mathrm{ej}}^{\infty}$ ranges from $850 \mathrm{~km} \mathrm{~s}^{-1}$ to $1200 \mathrm{~km} \mathrm{~s}^{-1}$, which is substantially higher than that shown in Figure 2 (from $690 \mathrm{~km} \mathrm{~s}^{-1}$ to $980 \mathrm{~km} \mathrm{~s}^{-1}$ ). However, the slope of the $v \mathrm{CDF}$ in this velocity range is only slightly flatter than that estimated from the potential given by (Xue et al. 2008; also see discussions in Sesana et al. 2007). Therefore, our conclusions made in Sections 4 and 5 on comparisons of the simulated $v \mathrm{CDF}$ (mainly the shape) with the estimated $v$ CDF here are not sensitive to the choice of the Galactic potential (see further discussions in Section 4.3).

Note here that the Galactocentric distances of the detected HVSs range from $25 \mathrm{kpc}$ to $130 \mathrm{kpc}$, which correspond to their travel time from the GC ranging from a few tens to $260 \mathrm{Myr}$.

\section{TIDAL BREAKUP OF BINARY STARS BOUND TO THE} $\mathrm{MBH}$

The progenitors of the detected HVSs are probably originated from disk-like stellar structures in the GC (e.g., the CWS disk) as demonstrated by Lu et al. (2010). These progenitors are likely to be bound to the central $\mathrm{MBH}$. In order to investigate whether the statistical distribution of the detected HVSs shown in Section 2 can be reproduced by the TBK mechanism, here we first explore the detailed physics of the tidal breakup of binary stars that initially bound to the central $\mathrm{MBH}$ as it is still elusive in the literature. Consider that the binary star is moving on an eccentric orbit bound to the $\mathrm{MBH}$ and injecting/migrating into the vicinity of the $\mathrm{MBH}$. For convenience, we denote the injecting/migrating stellar binary by the "stellar" binary with semimajor axis $a_{\mathrm{b}}$, eccentricity $e_{\mathrm{b}}$, and mass $m=m_{1}+m_{2}$ (where $m_{1}$ and $m_{2}$ are the masses of its two components, respectively). The center of mass of the stellar binary and the $\mathrm{MBH}$ also compose a binary system, which we denote by the "outer" binary with semimajor axis $a_{\text {out }}$ and eccentricity $e_{\text {out }}$.

\subsection{Three-body experiments}

We perform three-body experiments of dynamical interactions between stellar binaries and the central MBH. We use the code DORPI5 based on the explicit fifth(fourth)-order Runge-Kutta method (Dormand \& Prince 1980; Hairer et al. 1993) to integrate the set of the equations that control the three-body interactions between the stellar binary and the $\mathrm{MBH}$. We set the tolerance of the fractional energy error to be $10^{-9}$ for a single orbit integration. We have also checked that statistically our results are not affected by setting a smaller minimum tolerance of the energy error. The initial conditions set for the stellar and the outer binaries include (1) the masses of the two components of the stellar binary $\left(m_{1}\right.$ and $\left.m_{2}, m_{2} \leq m_{1}\right)$ and its mass ratio $q=m_{2} / m_{1} ;(2)$ the initial semimajor axis and eccentricity of the stellar binary $\left(a_{\mathrm{b}, \mathrm{i}}, e_{\mathrm{b}, \mathrm{i}}\right) ;(3)$ the initial semimajor axis and eccentricity of the outer binary $\left(a_{\text {out }, \mathrm{i}}, e_{\mathrm{out}, \mathrm{i}}\right)$; (4) the orbital orientations of both the stellar and outer binaries; and (5) the initial orbital phases of both the stellar and outer binaries.

For convenience, we also define a dimensionless penetration parameter $D$ to describe how close a stellar binary can approach the $\mathrm{MBH}$ relative to its tidal 


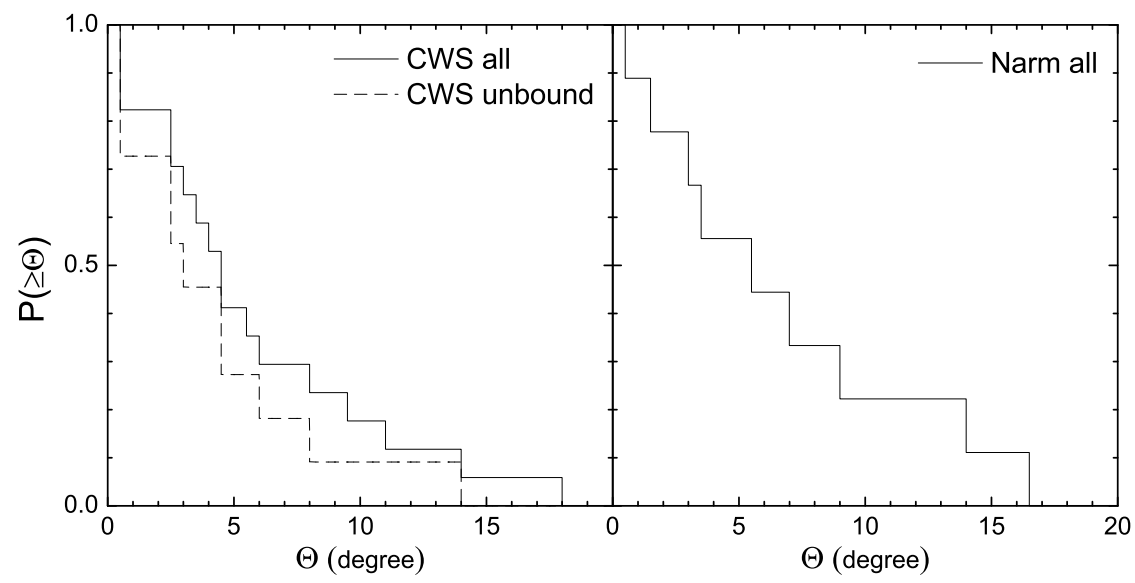

Fig. 1.- Observational inclination distributions of HVSs relative to their best-fit planes ( $\Theta C D F, P(\geq \Theta)$ ). The left panel is for the first population of HVSs which are spatially associated with a disk plane with an orientation similar to the CWS disk, and the right panel for the second population of the HVSs which are spatially associated with a disk plane with an orientation similar to the northern arm (Narm) of the minispiral or the warped outer part of the CWS disk. The solid histograms represent the distributions of all the detected HVSs, for each corresponding population, including the unbound HVSs, the bound HVSs and the HVS candidates. The distribution of the unbound HVSs is also shown for the first population by a dashed line. See Section 2.1

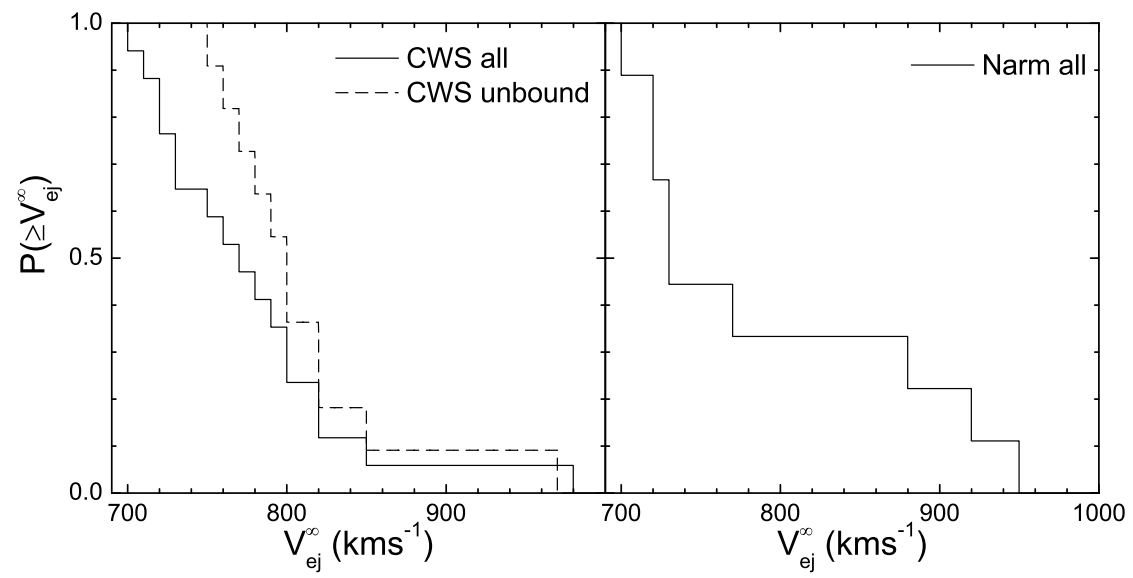

FIG. 2.- Observational cumulative distribution of the ejection velocity at infinity for the two populations of the detected HVSs $(v$ CDF, $\left.P\left(\geq v_{\mathrm{ej}}^{\infty}\right)\right)$. Other legends are the same as in Figure1 See Section 2.2

breakup radius $r_{\mathrm{tb}}$ (see a similar definition in Hills 1988. Bromley et al. 2006), i.e.,

$$
D \equiv 100 \frac{r_{\mathrm{p}, \mathrm{i}}}{r_{\mathrm{tb}}}=\frac{r_{\mathrm{p}, \mathrm{i}}}{a_{\mathrm{b}, \mathrm{i}}}\left[\frac{3 M_{\bullet}}{10^{6}\left(m_{1}+m_{2}\right)}\right]^{-1 / 3},
$$

where $r_{\mathrm{p}, \mathrm{i}}=a_{\mathrm{out}, \mathrm{i}}\left(1-e_{\mathrm{out}, \mathrm{i}}\right)$ is the initial pericenter distance and $r_{\mathrm{tb}}=\left[3 M_{\bullet} /\left(m_{1}+m_{2}\right)\right]^{1 / 3} a_{\mathrm{b}, \mathrm{i}}$. The penetration parameter $D=100$ corresponds to the tidal breakup radius of a stellar binary, and the range of $D$ of interest in this paper is $D \lesssim 250$ where tidal breakup of stellar binaries is possible.

\subsubsection{Initial settings}

For the numerical experiments in Sections 3.2 and 3.3 . we simply set the mass ratio of the stellar binary as $q=1$, and $m_{1}=m_{2}=3 M_{\odot}$; but for those in Section 4 we adopt more realistic distributions of the mass ratio and the masses of the two components of the stellar binaries according to current observations on binary stars.

We set the initial semimajor axis $a_{\mathrm{b}, \mathrm{i}}$ of the stellar binary in the range from $0.05 \mathrm{AU}$ to $2 \mathrm{AU}^{3}$ Perturbations on a binary star with $a_{\mathrm{b}, \mathrm{i}}<0.05 \mathrm{AU}$ from the

3 The timescale for those stellar binaries to be disrupted tidal field of the MBH easily lead to the merger of its two components; while tidal breakup of binary stars with $a_{\mathrm{b}, \mathrm{i}}>2$ AU usually leads to ejections of stars with velocities substantially smaller than the hypervelocities interested in this paper. For demonstration only, we simply choose $a_{\mathrm{b}, \mathrm{i}}=0.1 \mathrm{AU}$ and $e_{\mathrm{b}, \mathrm{i}}=0$ (or $0.1,0.3$, and 0.6 alternatively) in the three-body experiments presented in Sections 3.2 and 3.3. but in Section 4 , we adopt a distribution of $a_{\mathrm{b}, \mathrm{i}}$ based on the constraint obtained from current observations and $e_{\mathrm{b}, \mathrm{i}}=0$.

We set the semimajor axis of the outer binary as $a_{\text {out }, \mathrm{i}} \sim 0.04-0.5 \mathrm{pc}$ (Lu et al. 2009; Gillessen et al. 2009), as the stellar binaries are possibly originated from the CWS disk within half a parsec from the central $\mathrm{MBH}$. The pericenter distance of the binary is set to be close to the tidal radius of the stellar binary. In Sections 3.2 and 3.3 . we set the penetration parameter $D$ typically to be $\sim 20-250$ and choose $a_{\mathrm{out}, \mathrm{i}}=0.2 \mathrm{pc}$ for demonstration

by their interactions with background stars is roughly $10^{9} \mathrm{yr}$ Yu \& Tremaine 2003; Hopman 2009). If these binaries are rotating around the $\mathrm{MBH}$ with semimajor axis of $\lesssim 1 \mathrm{pc}$ as adopted in this paper, the total time for $\sim 1000$ orbits is roughly $\lesssim 10^{7} \mathrm{yr}$. Therefore, the evolution of stellar binaries due to their dynamical interactions with background stars can be safely ignored. 
only.

The orientation of the orbit of the stellar binary, relative to that of the outer binary, $\phi \in[0, \pi]$, is assumed to be uniformly distributed in $\cos \phi$, if not specified.

The initial orbital phases of both the stellar and outer binaries are randomly chosen in all the following calculations.

\subsubsection{Approximations}

As the stellar binaries are initially set on orbits weakly bound to the central MBH, binaries with large penetration parameters (e.g., $D \gtrsim 150$ ) may revolve around the central MBH for many (e.g., 10-100 or even 1000) orbits. In our calculations below, the period of the stellar binary is usually much smaller than that of the outer binary. Therefore, most of the calculation time may be spent on integrating the stellar binary orbit when it is faraway from the MBH. However, when the binary star is faraway from the $\mathrm{MBH}$, the tidal torque from the $\mathrm{MBH}$ on the binary star is rather weak, so we can approximate the motion of the stellar binary around the $\mathrm{MBH}$ into two independent two-body problems: one is for the stellar binary, and the other is for the outer binary on an elliptical orbit, both of which can be done analytically. We adopt the above two-body approximation when the tidal force from the MBH on the stellar binary is less than $10^{-6}$ of the gravitational force between its two components (we have checked the two-body approximation by setting a lower threshold, e.g., $10^{-7}$ or $10^{-8}$, and found no significant difference in our results). With this approximation, our calculation time is sped up substantially when $a_{\text {out }}$ is large $(\gtrsim 0.2 \mathrm{pc})$ and its accuracy can still be maintained.

A star may be tidally disrupted if its close passage to the $\mathrm{MBH}$ is $\lesssim r_{\text {tid }}^{*} \equiv R_{*}\left(M_{\bullet} / m_{*}\right)^{1 / 3}$, where $m_{*}$ and $R_{*}$ are the mass and radius of the star, respectively. The radius of a star can be roughly given by $R_{*} \propto R_{\odot}\left(m_{*} / M_{\odot}\right)$ for $m_{*}<M_{\odot}$ and $R_{*} \propto R_{\odot}\left(m_{*} / M_{\odot}\right)^{0.75}$ otherwise, where $R_{\odot}$ is the solar radius (Torres et al. 2010, and references therein). Due to the tidal disruption, part of the disrupted remnants may be swallowed by the central $\mathrm{MBH}$ and part may be ejected out. In our calculations, we terminate the evolution of the corresponding system once any component of the stellar binary approaches within a radius $r_{\text {tid }}^{*}$ from the $\mathrm{MBH}$.

The tidal torque from the MBH may change the semimajor axis and the eccentricity of the stellar binary during its close passage to the MBH (see further discussions in Section 3.2.1). In the three-body experiment, we assume that the two components of the binary star merge into a single star once the distance between the two components becomes smaller than the sum of their radii, and the evolution of the corresponding system is terminated once a merger event occurs.

The consequences on the dynamical interactions of stellar binaries with the MBH can be different depending on whether the stellar binaries are initially on bound or unbound orbits. If the binary star is initially bound to the $\mathrm{MBH}$, it may revolve around the central $\mathrm{MBH}$ for many orbits before its disruption or merger; while the binary initially unbound to the $\mathrm{MBH}$ passes by the $\mathrm{MBH}$ only once even if it is not broken up during its close passage (see further discussions in Sections 3.2 and 3.3). Multiple times of interactions of bound binary stars with a central $\mathrm{MBH}$ have been discussed by Antonini et al. (2010), in which they focus on the post-Newtonian effects on stellar orbits in the gravitational field of the $\mathrm{MBH}$ and mergers of the two components of the binary. In Section 3.2, we analyze the consequence of the first close encounter of the stellar binary with the central $\mathrm{MBH}$, and the changes in the orbital elements of the stellar binary (if the binary star survives). Then we consider the cumulative effects of multiple encounters in Section 3.3 . We focus on discussing how the spatial and velocity distributions of HVSs are connected with their originations, ejecting mechanisms, and dynamical environments in the GC.

In Section 3.2, $10^{4}$ three-body experiments are performed for each of the four set of initial conditions, i.e., $e_{\mathrm{b}, \mathrm{i}}=0,0.1,0.3$, and 0.6 , respectively. If a stellar binary is not tidally broken up during its first close passage to the $\mathrm{MBH}$, the three-body experiment ends up when the stellar binary reaches the apoapsis after its first close passage to the MBH. In Section 3.3, $10^{4}$ threebody experiments are performed for each set of initial conditions and those experiments that do not lead to disruption/merger/separation within 500 revolutions are excluded.

\subsection{Consequences of the first close passage of a stellar binary to the $M B H$}

Considering that binary stars are injected toward the $\mathrm{MBH}$ on weakly bound orbits with eccentricity close to 1 and $D \lesssim 250$, the changes in their orbits, as consequences of the tidal effect from the MBH, after their first close passages, can be characterized by the following five categories.

1. The two components of the stellar binary remain bound to each other, but its orbital parameters $\left(a_{\mathrm{b}}\right.$, $\left.e_{\mathrm{b}}\right)$ are changed by the tidal torque from the $\mathrm{MBH}$ during the close encounter. The outer binary is still bound and the stellar binary will encounter with the MBH again in its next close passage. The probability of our three-body experiments below resulted in this category is denoted by $P_{\text {bound }}$.

2. The binary star is tidally broken up, with one component of the binary being ejected out as a highvelocity star, and the other one being trapped onto an orbit that is tightly bound to the $\mathrm{MBH}$ compared to its parent stellar binary. The probability resulted in this category is denoted by $P_{\mathrm{ej}}$.

3. The binary is tidally broken up into two single stars, and the two stars are not bound to each other but both bound to the central $\mathrm{MBH}$. The probability resulted in this category is denoted by $P_{\mathrm{sp}}$.

4. The two components of the binary merge into one single star. The probability resulted in this category is denoted by $P_{\mathrm{mrg}}$.

5. One (or both) component(s) of the binary is (are) tidally disrupted and partly swallowed by the central MBH. The probability resulted in this category is denoted by $P_{\text {swallow }}\left(=1-P_{\text {bound }}-P_{\mathrm{ej}}-P_{\mathrm{sp}}-\right.$ $\left.P_{\mathrm{mrg}}\right)$. 


\subsubsection{Consequence 1}

If a stellar binary can still maintain its integrity after its close passage to the central MBH, the eccentricity and the semi-major axis of the binary are generally changed due to the tidal torque from the $\mathrm{MBH}$.

- Changes in eccentricities. Figure 3 shows the simulation results of the changes in eccentricities of the stellar binaries after their first close passages to the $\mathrm{MBH}$. When the penetration parameter is small (e.g., $D \lesssim 150$ ), the eccentricity of the stellar binaries may be excited (or de-excited) to various values in the range from 0 to 1 . The large range of the excited (or de-excited) eccentricities is mainly due to various phases of the stellar binaries at the close passages to the $\mathrm{MBH}$ and various orientations of the stellar binaries relative to the orbit of the outer binary. For encounters with $D \gtrsim 200$, excitation (or de-excitation) on eccentricities is relatively small, especially for those binaries with $e_{\mathrm{b}, \mathrm{i}}=0$. Multiple subsequent encounters of the stellar binary with the MBH should be common at $D \gtrsim 150$, where both $P_{\text {ej }}$ and $P_{\mathrm{mrg}}$ are small and $P_{\text {bound }}$ is large (see Figure 5). In these cases, the binary eccentricity (and semimajor axis) may then be further changed due to the cumulative effect of multiple subsequent encounters (see Section 3.3).

- Changes in semimajor axes. As shown in Figure 4 . the semimajor axis of a binary star can be excited to a larger value but can also be de-excited to a smaller value. The changes of the semimajor axes depend on the orbital phases of the stellar binary during its close encounter. If the relative positional vector of the two components of the stellar binary is aligned with the radial vector from the central MBH, the stellar binary is more likely to be stretched and its semimajor axis is changed to a larger value after the encounter; while the binary can also be shrunk and its semimajor axis may be changed to a smaller value if the relative positional vector of its two components is close to perpendicular to the radial vector. The time for the binary passing by the periapsis is comparable to, if not substantially smaller than, the orbital period of the stellar binary when the closest approach is roughly the tidal radius of the binary. Therefore, whether the binary is stretched or shrunk is determined by the orbital phase and orientation of the stellar binary during its close encounter with the central MBH. As the stellar binary is stretched in most of its orbital phases, the semimajor axis of the binary is more likely to be excited rather than de-excited after its first close passage to the $\mathrm{MBH}$ (see Figure 4). We also find that the relative change of the semimajor axis of the stellar binary is independent of the initial semimajor axis of the binary, which is verified by our calculations with adopting different $a_{\mathrm{b}, \mathrm{i}}$.

In addition, we note here that the orbit of the outer binary star almost remains the same after the first close encounter if the stellar binary can maintain its integrity. Our calculations above show that the semimajor axis of outer binaries may change by $\delta a_{\text {out }} \lesssim 0.02 a_{\text {out }}$ and the distance to the pericenter of the outer binary may only change by $\delta r_{\mathrm{p}} \lesssim 0.001 r_{\mathrm{p}}$, which suggests that the location of the subsequent close encounters is almost the same as that of the first encounter if no other perturbation takes place on the orbit of the binary star.

\subsubsection{Consequences 2 and 4: ejection and merging probabilities}

Figure 5] shows our calculation results on the ejection probability $P_{\mathrm{ej}}$ and the merging probability $P_{\mathrm{mrg}}$. As seen from Figure 5, $P_{\text {ej }}$ increases with decreasing penetration parameters $D$ because the tidal force from the $\mathrm{MBH}$ increases with decreasing $D$. And $P_{\text {ej }}$ also increases with increasing initial eccentricities of the stellar binaries. The reason is that the distance between the two components of a binary for given $D$ and $a_{\mathrm{b}}$, and hence the tidal torque on them, is larger if the binary has a larger eccentricity, and therefore the binary is easier to be broken up. For the same reasons, $P_{\text {mrg }}$ shown in Figure 5 decreases with increasing $D$ at the large- $D$ end, but decreases with decreasing $D$ at the low- $D$ end due to the significant increase of $P_{\text {ej }}$. A stellar binary with a larger initial eccentricity is also easier to be excited to an eccentricity high enough for its two components to merge and thus $P_{\mathrm{mrg}}$ increases with increasing $e_{\mathrm{b}, \mathrm{i}}$ at large- $D$ end.

Figure 6 shows the ejection velocity at infinity $\left(v_{\mathrm{ej}}^{\infty}\right)$ of the ejected component as a function of the penetration parameter $(D)$ of stellar binaries with different initial eccentricities $\left(e_{\mathrm{b}, \mathrm{i}}=0,0.1,0.3\right.$, and 0.6 , respectively). We find that the rms values of the ejection velocities can be well fitted by the following formula:

$$
\begin{aligned}
\left\langle\left(v_{\mathrm{ej}}^{\infty}\right)^{2}\right\rangle^{1 / 2}= & \frac{v_{\mathrm{ej}, 0}^{\infty}}{\sqrt{1+e_{\mathrm{b}, \mathrm{i}}}}\left[1-\left(\frac{D_{\mathrm{eff}}}{260}\right)^{2}\right]\left(\frac{a_{\mathrm{b}, \mathrm{i}}}{0.1 \mathrm{AU}}\right)^{-1 / 2} \times \\
& \left(\frac{m_{1}+m_{2}}{6 M_{\odot}}\right)^{1 / 3}\left(\frac{M_{\bullet}}{4 \times 10^{6} M_{\odot}}\right)^{1 / 6},
\end{aligned}
$$

for $20<D<260$, where $D_{\text {eff }}=D / \sqrt{1+e_{\mathrm{b}, \mathrm{i}}}$ is the effective penetration parameter for a stellar binary with non-zero eccentricity, and $v_{\mathrm{ej}, 0}^{\infty}=2560 \mathrm{~km} \mathrm{~s}^{-1}$ is the normalization of the ejection velocity when $e_{\mathrm{b}, \mathrm{i}}=0$ and $a_{\mathrm{b}, \mathrm{i}}=0.1$ AU. For stellar binaries with zero initial eccentricities and any $D$ in the range of $20-150$, this fitting formula gives a value similar to that given by Equation (1) in Bromley et al. (2006).

If $a_{\text {out, } i}$ is set to a smaller value, the ejection velocity may be changed by $v_{\mathrm{ej}}^{\infty} \rightarrow \sqrt{\left(v_{\mathrm{ej}}^{\infty}\right)^{2}-G M_{\bullet} / a_{\mathrm{out}, \mathrm{i}}}$ because the ejected star has to first overcome its initial bounding energy. If $a_{\text {out,i }}=0.01 \mathrm{pc}$, for example, the ejection probability $P_{\mathrm{ej}}$ is significantly smaller than that shown in Figure [5] and the ejection velocity $v_{\mathrm{ej}}^{\infty}$ is also substantially smaller because a significant fraction of the stellar binaries end up in consequence 3 instead of consequence 2 .

\subsection{Multiple encounters}

3.3.1. The ejection/merging probabilities and the number of revolutions 


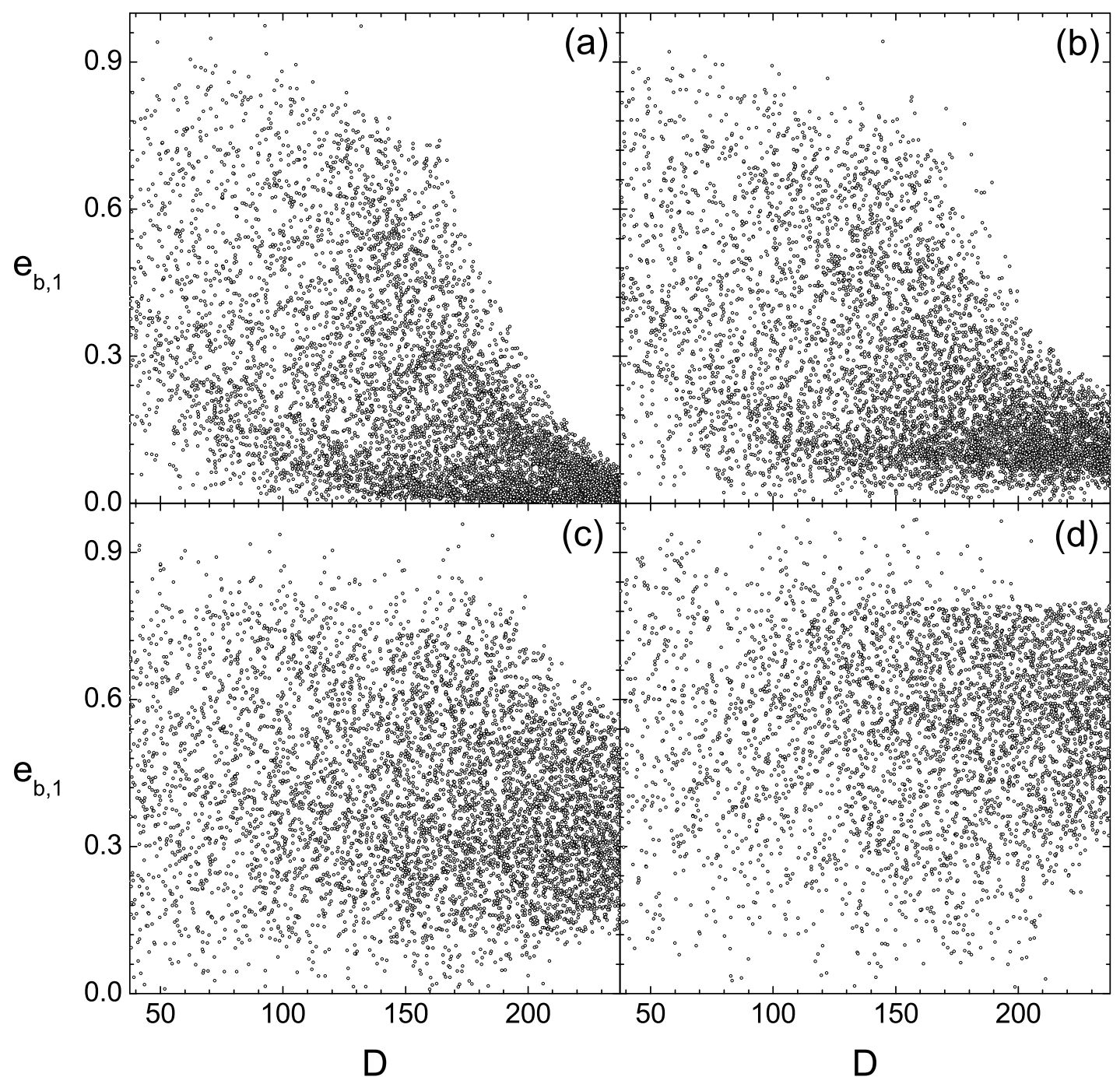

FIG. 3.- Eccentricity of the binary stars after their first close encounters with the central MBH $\left(e_{\mathrm{b}, 1}\right)$ vs. the penetration parameter $D$, if the binary star survives. The semimajor axes and the masses of the binaries are all set to be $a_{\mathrm{b}, \mathrm{i}}=0.1 \mathrm{AU}$ and $m_{1}=m_{2}=3 M_{\odot}$, respectively. The semimajor axes of the outer binaries are all set to be $a_{\text {out, } \mathrm{i}}=0.2 \mathrm{pc}$. Panels (a)-(d) show the $e_{\mathrm{b}, 1}$ for those stellar binaries with initial eccentricities of $e_{\mathrm{b}, \mathrm{i}}=0,0.1,0.3$, and 0.6 , respectively. As seen from this figure, the eccentricity of a binary may change to various values between 0 and 1 after the first close encounter if $D<150$. The total numbers of survived binary stars in the $10^{4}$ three-body experiments here are 7256, 7160, 6709, and 4782 in panels (a), (b), (c), and (d), respectively.

The tidal effect from the $\mathrm{MBH}$ may be not large enough to break up a stellar binary with a large $D$ (e.g., $D \gtrsim 150)$ during the first close passage of the binary. If the binary star is initially bound to the $\mathrm{MBH}$, multiple times of close passages of the binary to the $\mathrm{MBH}$ should be common, and thus the probabilities of ejection and stellar coalescence are substantially enhanced, especially at large $D$, as demonstrated in Figure 7 , which can be understood as follows.

- The increase of the number of close passages to the $M B H$. Assuming that the ejection probability after each close passage of the binary is roughly the same as denoted by $P_{\text {ej,0 }}$ and the probability of the binary maintaining its integrity is roughly $P_{\text {bound,0, }}$ then after $N_{\text {orb }}$ revolutions the cumulative ejection probability is given by

$$
P_{\mathrm{ej}} \simeq \sum_{i=1}^{N_{\text {orb }}} P_{\mathrm{ej}, 0} P_{\mathrm{bound}, 0}^{i-1}=P_{\mathrm{ej}, 0} \frac{1-P_{\mathrm{bound}, 0}^{N_{\text {orb }}}}{1-P_{\mathrm{bound}, 0}}
$$

If $N_{\text {orb }} \rightarrow \infty$, then $P_{\text {ej }}=P_{\text {ej }, 0} /\left(1-P_{\text {bound }, 0}\right)>$ $P_{\text {ej }, 0}$. When $D$ is large $(\gtrsim 150), P_{\text {ej }}$ can increase substantially with increasing $N_{\text {orb }}$ as $P_{\text {bound,0 }}$ is close to 1 .

- The increase of the semimajor axis of the stellar binary. The semimajor axis of the stellar binary may be excited to be larger than its initial value after the close passages (see Figure 6), and correspondingly the penetration parameter $D \propto r_{\mathrm{p}} / a_{\mathrm{b}}$ decreases as $r_{\mathrm{p}}$ is almost not changed. A smaller $D$ results in a larger $P_{\text {ej }}$. As demonstrated in Figure 8, the semimajor axes of many stellar binaries 


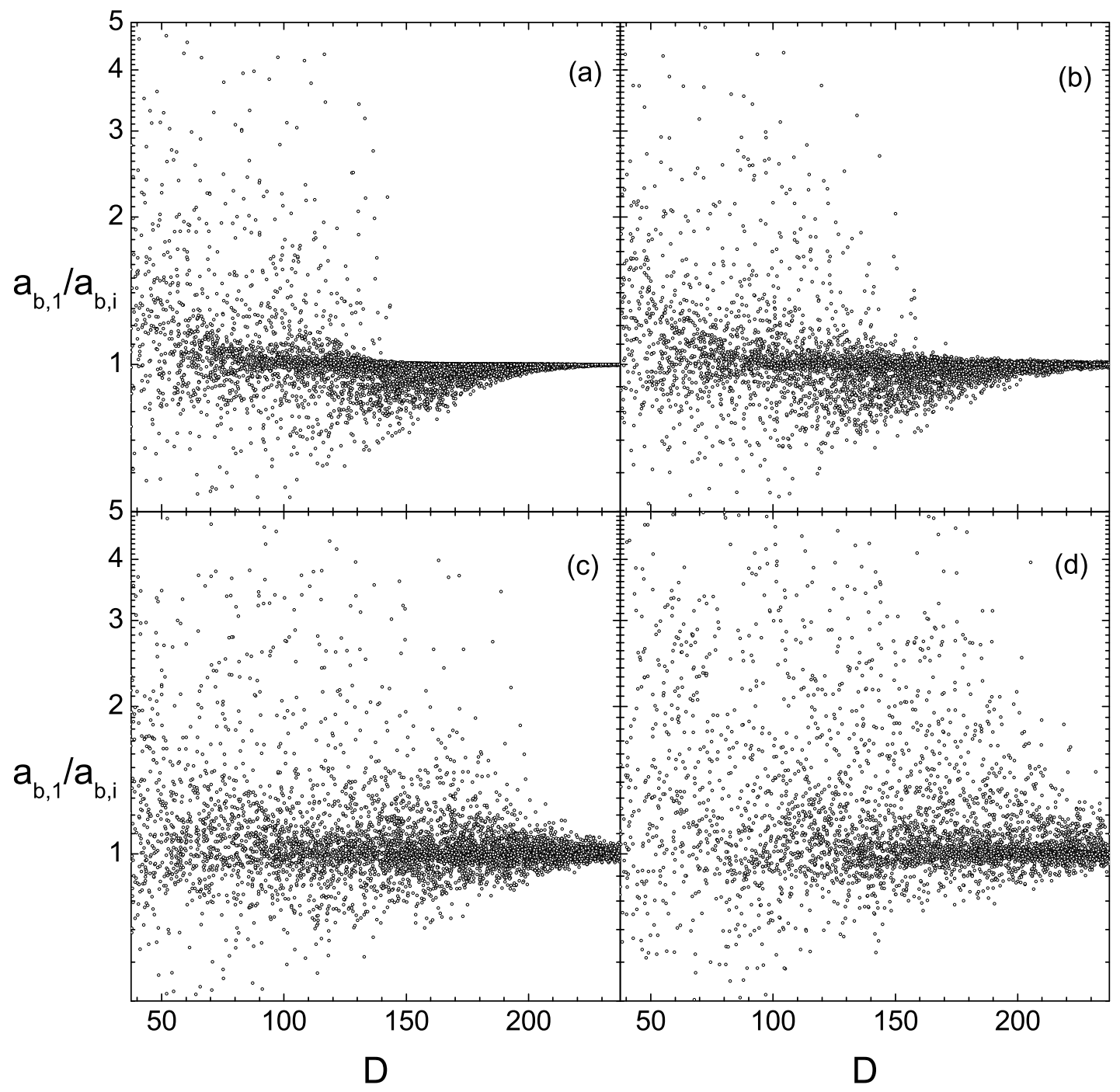

FIG. 4.- Relative changes in the semimajor axes of the binary stars after their first close passages to the central MBH as a function of the penetration parameter $D$. The initial conditions for the stellar binaries are the same as that in Figure 3 The initial eccentricities of the stellar binaries are $e_{\mathrm{b}, \mathrm{i}}=0$ (panel (a)), 0.1 (panel (b)), 0.3 (panel (c)), and 0.6 (panel (d)), respectively. Generally, the change in $a_{\mathrm{b}}$ is large if $D$ is small. The total number of survived binary stars shown in each panel is the same as that in Figure 3

before their breakup and last close encounters with the $\mathrm{MBH}$ are substantially larger than their initial values. As the tidal force is approximately a monotonically increasing function of $a_{\mathrm{b}}$, the semimajor axes of many binaries tend to rapidly (exponentially) increase after many subsequent close encounters with the $\mathrm{MBH}$, while the semimajor axes of some other binaries decrease only slightly, which leads to the asymmetric distribution of $a_{\mathrm{b}, \mathrm{f}} / a_{\mathrm{b}, \mathrm{i}}$, i.e., the relative change in $a_{\mathrm{b}}$ for many binaries with $a_{\mathrm{b}}$ excited is substantially larger than that for a relatively small number of binaries with $a_{\mathrm{b}}$ de-excited, as shown in Figure 8 .

- The increase of the eccentricity of the stellar binary. Binary stars with large penetration parameters $(D \gtrsim 150)$ may be hard to break up initially. However, their eccentricities are relatively easy to be excited to large values due to cumulative tidal effects from the central $\mathrm{MBH}$ during their multiple encounters with the MBH. The increase in the eccentricity thus leads to a larger ejection or merging probability as mentioned in Section 3.2. We note here that the Kozai mechanism may cause periodical oscillations of the internal eccentricity of stellar binaries during their multiple encounters with the $\mathrm{MBH}$, which may be important in enhancing the merger of the two components of those binaries. A detailed study of this resonance can be found in Antonini et al. (2010).

For those stellar binaries injected into the vicinity of the $\mathrm{MBH}$ with $D \sim 200-250, \sim 90 \%$ of them are disrupted or merged within 500 revolutions. For those stellar binaries that are broken up within 500 revolutions with one component being ejected out, Figure 9 shows the cumulative probability distribution of the number of revolutions around the $\mathrm{MBH}\left(N_{\text {orb }}\right)$ that they experienced. The majority of the stars are broken up within 10 revolutions when $D$ is small, while most of them can be 


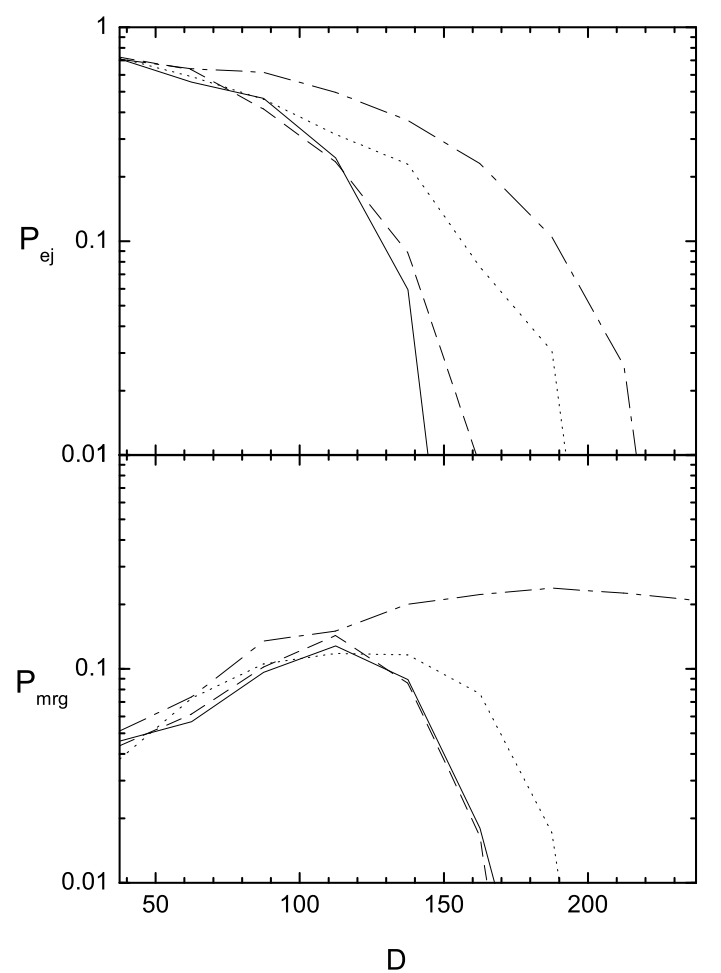

FIG. 5.- Ejection and merging probabilities $\left(P_{\mathrm{ej}}\right.$ and $\left.P_{\mathrm{mrg}}\right)$ of the stellar binaries after their first close passages to the $\mathrm{MBH}$ as a function of the penetration parameter $D$ (top panel and bottom panel, respectively). The initial conditions set for the stellar binaries are the same as those in Figure 3 The solid, dashed, dotted, and dot-dashed curves represent the results for those stellar binaries with initial eccentricities $e_{\mathrm{b}, \mathrm{i}}=0,0.1,0.3$, and 0.6 , respectively.

broken up only after many revolutions when $D$ is large. If $250>D>200$, for example, $N_{\text {orb }}$ ranges from 4 to 500 (see Figure 9), and about $40 \%$ of those stellar binaries are broken up after 100 encounters. We should note that the ejection velocities of the resulted HVSs at large $D$ are substantially smaller than those of the stellar binaries broken up at small $D$.

\subsubsection{The effects of relative orientations}

The tidal effects on a stellar binary during its close passages to the central MBH are also affected by the initial orientation of the stellar binary orbit with respect to the outer binary orbit. Given the penetration parameter $D$, the prograde rotating stellar binary is easier to be tidally broken up and can receive a larger kick in velocity during the close passages than a retrograde rotating stellar binary. Compared to prograde rotating stellar binaries, retrograde rotating binaries can revolve around the central $\mathrm{MBH}$ for many more orbits before they are broken up. And the ejection velocities of the stars from the interaction of retrograde binaries with the $\mathrm{MBH}$ are also much smaller than that for the cases of prograde binaries. In Table 1, we list some numerical results to show the effect of different relative orientations of the stellar binary with respect to that of the outer binary. (For demonstration, only the results from those experiments with the stellar binaries initially having $a_{\mathrm{b}, \mathrm{i}}=0.1 \mathrm{AU}$, $e_{\mathrm{b}, \mathrm{i}}=0$, and $D=100$ are shown in Table 1)
TABLE 1

EFFECTS OF THE RELATIVE ORIENTATION OF THE STELLAR BINARY ORBIT TO THE OUTER BINARY ORBIT

\begin{tabular}{lcccccc}
\hline \hline Orientation & $P_{\mathrm{ej}}$ & $P_{\mathrm{mrg}}$ & $P_{\mathrm{sp}}$ & $\sqrt{\left\langle v_{\mathrm{ej}}^{\infty 2}\right\rangle^{\mathrm{a}}}$ & $\frac{\sigma}{\sqrt{\left\langle v_{\mathrm{ej}}^{\infty}{ }^{2}\right\rangle}}$ & $\left\langle N_{\mathrm{orb}}\right\rangle^{\mathrm{c}}$ \\
\hline Prograde & 0.88 & 0.12 & 0.000 & 2.39 & +0.13 & 1.1 \\
Retrograde & 0.66 & 0.29 & 0.038 & 1.17 & ${ }_{-0.15}^{+0.53}$ & 16 \\
Uniform & 0.86 & 0.13 & 0.004 & 1.80 & ${ }_{-0.37}^{+0.27}$ & 3.9 \\
\hline
\end{tabular}

Note. - In the first column, "prograde" (or "retrograde") denotes that all the stellar binaries are rotating in the same direction as (or the anti-direction of) the outer binary, while "uniform" denotes that the orientations of the stellar binaries with respect to that of the outer binary $\phi$ are uniformly distributed in $\cos \phi$ for $\phi \in[0, \pi]$. This table shows the results from those experiments with the stellar binaries initially having $a_{\mathrm{b}, \mathrm{i}}=0.1 \mathrm{AU}, e_{\mathrm{b}, \mathrm{i}}=0$, and $D=100$.

a Mean ejection velocity at infinity in units of $10^{3} \mathrm{~km} \mathrm{~s}^{-1}$.

b Errors in the rms of the ejection velocities at infinity.

c Mean number of close encounters experienced by the stellar binaries before their breakup and the ejection of one of its two components (i.e., the consequence 2 in Section 3.2.

TABLE 2

Different MODELS FOR THE TBK MECHANISM

\begin{tabular}{ccccc}
\hline \hline Model & $f\left(a_{b, i}\right)^{\mathrm{a}}$ & $f\left(r_{\mathrm{p}}\right)$ & $a_{\text {out }, \mathrm{i}}(\mathrm{pc})$ & $v_{\text {ini }}^{\infty}\left(\mathrm{km} \mathrm{s}^{-1}\right)^{\mathrm{b}}$ \\
\hline UB-1 & Öpik & Constant & $\ldots$ & 250 \\
UB-2 & log-N & Constant & $\ldots$ & 250 \\
LP-1 & Öpik & Constant & $0.04-0.5$ & $\ldots$ \\
LP-2 & log-N & Constant & $0.04-0.5$ & $\ldots$ \\
RW-1 & Öpik & Random walk & $0.04-0.5$ & $\ldots$ \\
RW-2 & log-N & Random walk & $0.04-0.5$ & $\ldots$ \\
\hline
\end{tabular}

a The "log-N" denotes those models adopting a log-normal distribution of $f\left(a_{\mathrm{b}, \mathrm{i}}\right)$ as that given by Duquennoy \& Mayor (1991), and the "Öpik" denotes those models adopting the Öpik law for $f\left(a_{\mathrm{b}, \mathrm{i}}\right)$.

b For the "UB" model, those stellar binaries initially have velocities of $250 \mathrm{~km} \mathrm{~s}^{-1}$ at infinity.

\subsubsection{Deflection angles}

Here, we briefly summarize the results about the deflection angles between the direction of an HVS moving away from the Galactic halo and the initial injecting direction of its progenitorial binary. The ejecting direction of an HVS is almost anti-parallel to the injecting direction of its progenitor and the off-set $\delta \Theta$ is $\lesssim 15^{\circ}$, as demonstrated by Lu et al. (2010, see Figure 1). In another words, HVSs can well memorize the injecting directions of their progenitors. We also find here that the major component of $\delta \Theta$ is in the orbital plane of the outer binary and the other component of the off-set, which is perpendicular to the orbital plane of the outer binary and is relatively insignificant $\sim 1^{\circ}-2^{\circ}$. The main reason for this result is that the stretching on the stellar binary by the MBH tidal force is most effective when the orientation of the stellar binary is similar to that of the outer binary orbit. Considering the gravitational potential due to the stellar cusp surrounding the MBH (which currently seems not to be clearly determined; (Do et al. 2009; Bartko et al. 2010)), the orbit of a stellar binary revolving around the $\mathrm{MBH}$ may precess significantly if the binary is broken up only after more than hundreds of revolutions. Due to this precession, the ejecting direction of a resulted HVS may deviate significantly from the injecting direction of its progenitor and the main compo- 


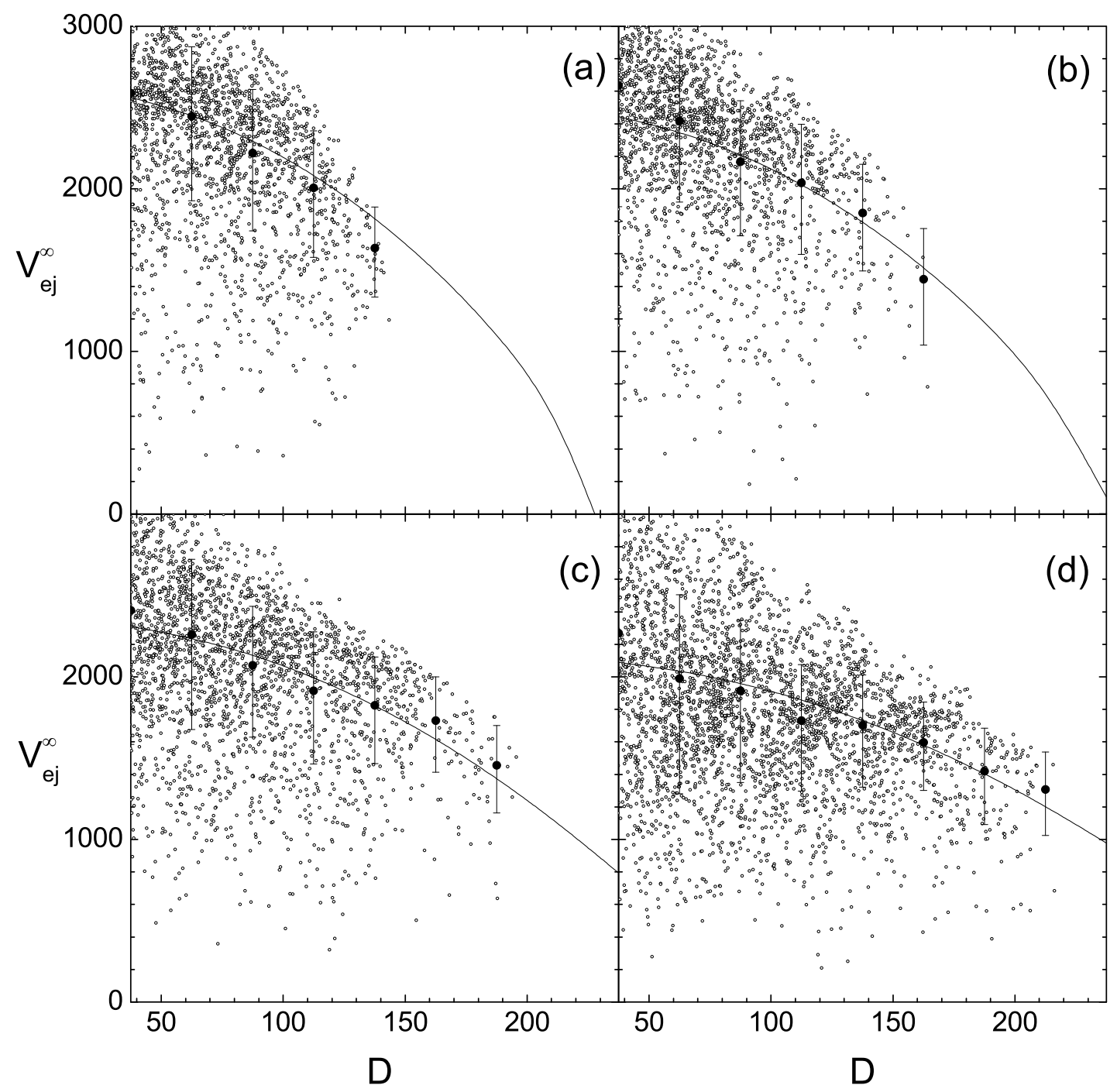

FIG. 6.- Ejection velocity at infinity $\left(v_{\mathrm{ej}}^{\infty}\right)$ for the ejected components of those stellar binaries that are tidally broken up after their first close passages to the central MBH. The initial conditions for the stellar binaries are the same as that in Figure 3 Panels (a), (b), (c), and (d) show the results for those stellar binaries with initial eccentricities $e_{\mathrm{b}, \mathrm{i}}=0,0.1,0.3$, and 0.6 , respectively. The filled circles are the square roots of the rms values of $\left(v_{\mathrm{ej}}^{\infty}\right)^{2}$ in different bins of penetration parameters $D$, and the error bars indicate the square roots of the standard deviations of $\left(v_{\mathrm{ej}}^{\infty}\right)^{2}$. The solid curves show the best fits to the rms values. The total numbers of the experiments shown are 2261, 2339, 2687, and 3543 in panels (a), (b), (c), and (d), respectively.

nent of the deviation is in the orbital plane of the outer binary. If projecting these two directions onto the plane that is perpendicular to the orbital plane of the outer binary, however, the difference between the two projected directions is still insignificant. This suggests that the spatial distribution of the ejecting HVSs can still maintain on the plane that their progenitors originated even if the precession due to the stellar cusp is significant. Therefore, the spatial distribution of HVSs should reflect the geometrical structure of the parent populations of their progenitorial binaries. If the progenitors of HVSs are originated from a disk-like structure, particularly, the resulted HVSs projecting to the infinity on the sky should locate close to a great circle with the same orientation as the disk (see Figure 5 in Lu et al. 2010). We will demonstrate this further in Section 4

\section{INCLINATION AND VELOCITY DISTRIBUTIONS OF HVSS FROM THE TBK MECHANISM}

\subsection{Initial settings}

To investigate the spatial and velocity distributions of HVSs, we now choose more realistic distributions of the parameters for both the stellar and outer binaries (i.e., initial conditions) involving in our numerical simulations as follows.

For the stellar binary, the initial conditions are set as follows under the assumption that they are originated from stellar disk(s) like the CWS one, though many important issues related to the disk origination of HVSs (such as warping and relaxation of the disk(s) and many young stars observed in the GC but not in the CWS disk; Lu et al. 2009; Bartko et al. 2010) need to be further investigated (Lu et al.|2010). (1) The distribution of 


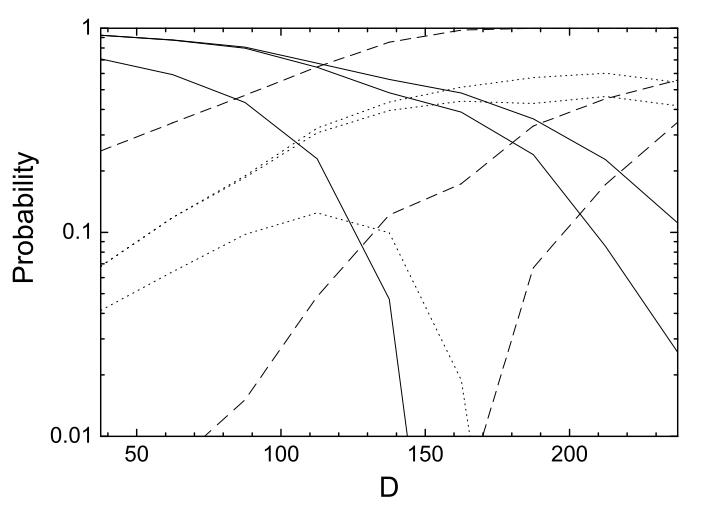

FIG. 7.- Probabilities of different consequences of binary stars after $N_{\text {orb }}$ times close passages to the central $\mathrm{MBH}$ as a function of the initial penetration parameter $D$. The solid, the dotted, and the dashed curves represent $P_{\mathrm{ej}}, P_{\mathrm{mrg}}$, and $P_{\mathrm{bound}}$, respectively (see Section 3.2). For each type of the curves, we show the results for $N_{\text {orb }}=1,20$, and 500, respectively (solid and dotted curves: from bottom to top; dashed curves: from top to bottom).

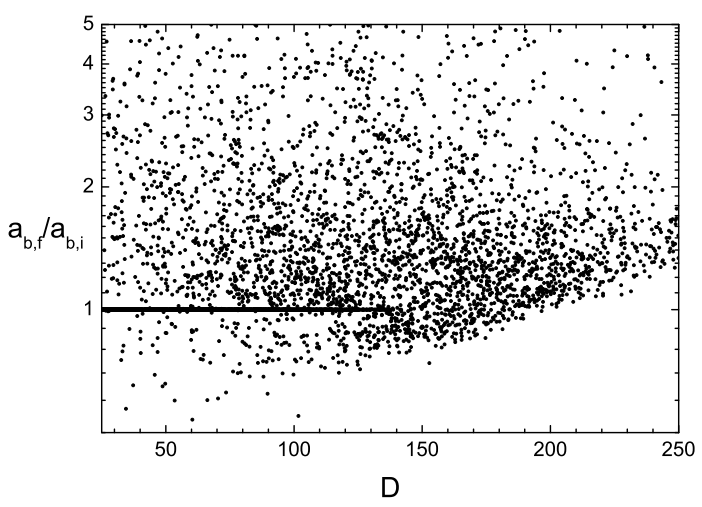

Fig. 8.- Ratio of the semimajor axis of the stellar binary to its initial value $\left(a_{\mathrm{b}, \mathrm{f}} / a_{\mathrm{b}, \mathrm{i}}\right)$ vs. the penetration parameter $D$, where $a_{\mathrm{b}, \mathrm{f}}$ is the semimajor axis of the stellar binary when it is at the apocenter of the last outer binary orbit just before its breakup. For those stellar binaries with $D>150$, most of them have $a_{\mathrm{b}, \mathrm{f}}$ substantially larger than $a_{\mathrm{b}, \mathrm{i}}$. In the figure, the total number of the stellar binaries that are broken up is 6007, of which 2116 are broken up after their first close passages to the $\mathrm{MBH}$ and thus have $a_{\mathrm{b}, \mathrm{f}} / a_{\mathrm{b}, \mathrm{i}}=1$.

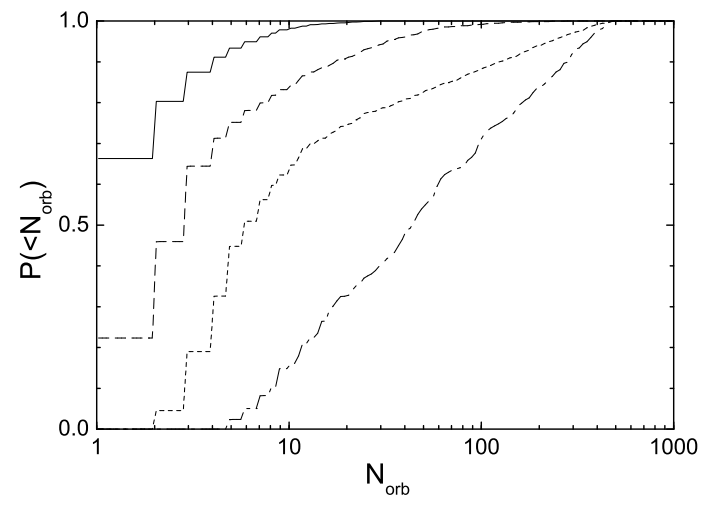

FIG. 9.- Cumulative probability distributions of the number of close passages that the stellar binaries have experienced before their breakup with ejecting one of their two components. Only those stellar binaries that are broken up within 500 revolutions around the $\mathrm{MBH}$ are taken into account. The solid, dashed, dotted, and dot-dashed lines represent the distributions with different ranges of penetration parameters $25<D<100,100<D<150,150<$ $D<200$, and $200<D<250$, respectively. their semimajor axes $a_{\mathrm{b}, \mathrm{i}}$ follows either the Öpik law, i.e., $P\left(a_{\mathrm{b}}\right) d a_{\mathrm{b}} \propto d a_{\mathrm{b}} / a_{\mathrm{b}}$ (e.g., Kobulnickv \& Frver 2007), or a log-normal distribution (Duquennoy \& Mayor 1991). (2) The mass distribution of the primary stars $m_{1}$ follows the Miller-Scalo initial mass function, i.e., $f\left(m_{1}\right) \propto$ $m_{1}^{-\alpha}$ and $\alpha \sim 2.7$ (e.g., Kroupa 2002). ${ }^{4}$ For massive binary stars, the distribution of the secondary star or the mass ratio $q=m_{2} / m_{1}$ can be described by two populations: (a) a twin population, i.e., about $40 \%$ binary stars have $q \sim 1$ and (b) the rest binaries, which follow a distribution of $f(q) \sim$ constant Kobulnicky \& Frver 2007; Kiminki et al. 2008, 2009). (3) The initial eccentricity of the stellar binary is assumed to be 0 , as adopted in some previous works (e.g., Bromley et al. (2006); Antonini et al. (2010)). (4) The orientation of the stellar binary orbital plane is chosen to be uniformly distributed in $\cos \phi$ for $\phi \in[0, \pi]$.

For the outer binary, the initial conditions are set as follows. (1) The orientation of the outer binary is set to satisfy a Gaussian distribution around the central planes of the stellar disks that initially hosted the stellar binaries with a standard deviation of $\sim 7^{\circ}-13^{\circ}$, (c.f., Lu et al. 2009; Bartko et al. 2009). The orientations of the host disks are assumed to be the same as that of the two best-fit planes, i.e., $(l, b)=\left(311^{\circ},-14^{\circ}\right)$ and $\left(176^{\circ}\right.$, $\left.-53^{\circ}\right)$, respectively (Lu et al. 2010). (2) The stellar binaries are initially set on orbits with semimajor axes of $a_{\text {out }, \mathrm{i}} \sim 0.04-0.5 \mathrm{pc}$, and this range is adopted according to the observational extents of the CWS disk. We note here that the extents of the structure associated with the second plane are not clear. Nevertheless, we assume that it is in the same range as the CWS disk. Adopting a slightly larger range (say, from $0.04 \mathrm{pc}$ to $1 \mathrm{pc}$ ) does not affect the results presented in this section and next section. (3) The distribution of $a_{\text {out,i }}$ of these binaries is assumed to follow the same surface density distribution as that of stars in the CWS disk $f\left(a_{\text {out,i }}\right) \sim a_{\text {out,i }}^{-2.3}$ (Lu et al.]2009; Bartko et al. 2009). (4) The distribution of the initial periapsis of the stellar binaries should depend on detailed mechanisms leading to the injection. It is not clear how bound binaries (in the outer disk region) are delivered to the immediate vicinity of the central MBH. Nevertheless, the mechanism responsible for the small orbital angular momentum of the stellar binary may fall into the two extreme categories discussed below.

- Large perturbations on the orbital angular momenta of binaries initially rotating around the $\mathrm{MBH}$ with insignificant eccentricities: in this scenario, changes of the orbital specific angular momenta of outer binaries are large compared with the specific angular momentum required to approach the tidal radius $r_{\mathrm{tb}}$ (denoted by $J_{\max } \simeq$ $\left.\sqrt{2 G M_{\bullet} r_{\mathrm{tb}}}\right)$, which leads to an even distribution of the distance to the periapsis of the outer binaries, i.e., $f\left(r_{\mathrm{p}}\right) \propto$ constant. Therefore, many binaries can penetrate into the vicinity of the $\mathrm{MBH}$ with small $D$, and some of them are broken up during the first close passage but others may be broken up or merged after many revolutions. Hereafter

${ }^{4}$ We have tested that adopting a top-heavy initial mass function as that in Bartko et al. (2010) does not significantly affect the resulted spatial and velocity distributions. 
the model adopting $f\left(r_{\mathrm{p}}\right) \propto$ constant for initially bound binaries with the above settings is denoted by the "LP" model. The distribution of $r_{\mathrm{p}}$ above is similar to that adopted for initially unbound stellar binaries which is discussed in Sesana et al. (2007). For comparison, we also introduce a model (denoted by "UB" below) in which the stellar binaries are initially unbound to the $\mathrm{MBH}$, similar to that used in Sesana et al. (2007).

- The perturbations on the orbital specific angular momenta of binary stars are small compared with $J_{\max }$, and $f\left(r_{\mathrm{p}}\right)$ is significantly different from that for the "LP" model. In this scenario, the eccentricity of an outer binary (or $r_{\mathrm{p}}$ ) may increase (or decrease) slowly. For example, Madigan et al. (2009) demonstrated that a secular instability of eccentric stellar disks rotating around the $\mathrm{MBH}$ can gradually excite some (binary) stars to extremely eccentric orbits $(e \rightarrow 1)$. The time period for this secular evolution can be hundred to thousand times of the orbital period of the outer binary before the breakup or merging of the stellar binary. Therefore, the probability for the stellar binary to be broken up is substantially even at a large $D$ due to the cumulative tidal effect from the $\mathrm{MBH}$ before the binary can possibly migrate into the region with a substantially small $D$ (as also illustrated in Figure 6). Note that the ejection velocity of the component escaping away from the MBH is substantially small if the binary is broken up at a large $D$ rather than at a small $D$. For these cases, the velocity distribution of the resulted HVSs may be substantially steeper than that from the LP model simply due to the suppression of the number of stellar binaries with small $D$. The detailed distribution of $f\left(r_{\mathrm{p}}\right)$ should depend on the detailed perturbations. Below we adopt a toy model for this scenario to obtain the spatial and velocity distributions of HVSs and compare them with that obtained from the "LP" model.

The orbit of the outer binary may be scattered by weak encounters of the binary star with other stars (in or out of the stellar disk), and its angular momentum $|J|$ may also be changed after each revolution. We approximate this as a one-dimensional diffusion process of $|J|$, and the standard deviation of the change of the angular momentum after each revolution $\sqrt{\left\langle(\delta|J|)^{2}\right\rangle}=$ constant $\ll|J|$. This random walk in $|J|$ corresponds to a change in $r_{\mathrm{p}}$ (i.e., $\left.\delta r_{\mathrm{p}}= \pm \xi \sqrt{r_{\mathrm{p}} / r_{\mathrm{p}, 0}}\right)$ after each revolution. The $r_{\mathrm{p}, 0}$ should be set by the penetration parameter (e.g., $D=250$ ) where the tidal effect begins to be important for a stellar binary with the maximum semimajor axis considered in this paper $\left(a_{\mathrm{b}, \mathrm{i}}=2 \mathrm{AU}\right)$. The periapsis of some stellar binaries may then diffuse inward to the vicinity of the MBH slowly. During this diffusion process, these stellar binaries may be broken up at a large $D$ after many revolutions and few stellar binaries with small $a_{\mathrm{b}, \mathrm{i}}$ can move into the very stellar region with small $D$. We denote this model as the "RW" model. For illustration, we set the model parameter $\xi=0.4 \mathrm{AU}$ and

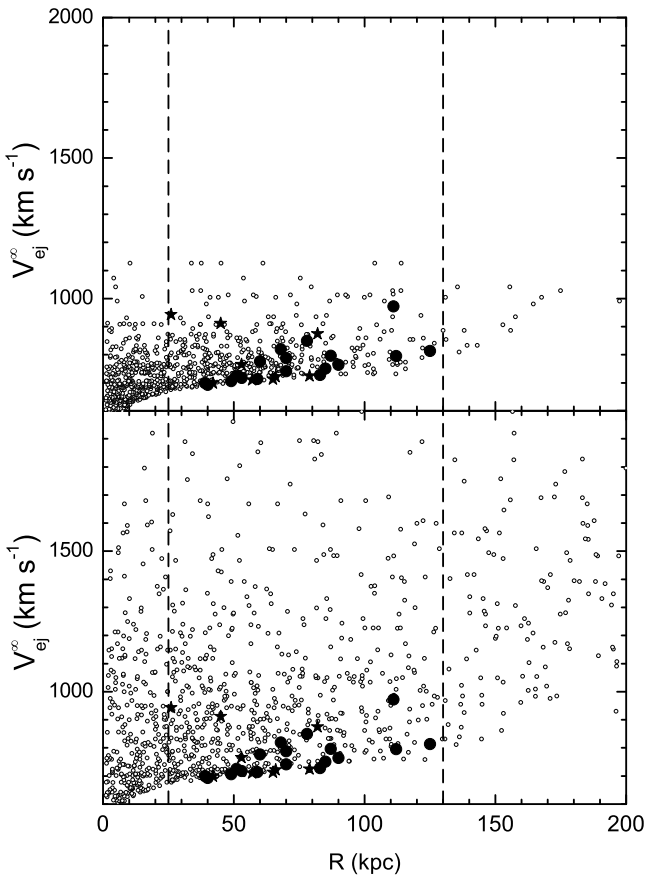

FIG. 10.- Distribution of simulated HVSs in the $v_{\mathrm{ej}}^{\infty}-R$ plane $(R$ is the Galactocentric distance of an HVS). The top and the bottom panels show the results obtained from the "RW1" model and the "LP1" model, respectively. The filled circles and stars represent the detected HVSs that are spatially associated with the best-fit plane with an orientation almost the same as that of the CWS disk and the best-fit plane with an orientation similar to that of the northern arm of the minispiral in the GC (or the outer warped part of the CWS disk), respectively. The dots represent the simulated HVSs. The apparent lower boundary is due to the selection effects of HVS candidates in observations (i.e., the cutoff at the radial velocity $v_{\mathrm{rf}}=275 \mathrm{~km} \mathrm{~s}^{-1}$ ). The vertical dashed lines are the lower and upper boundary of the distance of the detected HVSs from the GC. The total number of the simulated HVSs shown in the top and the bottom panel are 606 and 449, respectively. This figure shows that the ejection of HVSs with velocities $>1000 \mathrm{~km} \mathrm{~s}^{-1}$ is significantly suppressed in the "RW1" model.

$r_{\mathrm{p}, 0}=(D / 100) r_{\mathrm{tb}} \sim 630$ AU with $a_{\mathrm{b}}=2$ AU. A model with a much larger $\xi$ will be reduced to the "LP" model above.

Table 2 lists the above models and the settings of a few related parameters. According to the models (i.e., the "UB" model, the "LP" model, and the "RW" model), we use Monte Carlo simulations to obtain both the $\Theta C D F$ and the $v \mathrm{CDF}$. For each model, the total number of the three-body experiments is $10^{4}$. We only record those cases in which the masses of ejected stars are in the mass range $\left(3 M_{\odot}, 4 M_{\odot}\right)$ of the detected HVSs if not specified, and then calculate both the distribution of the inclination angles with respect to the central planes of their parent population and the velocity distributions.

To compare with the observations summarized in Section 2 the selection effects must be carefully considered. As shown in Figure 10, most HVSs are detected in Galactocentric distances from $25 \mathrm{kpc}$ to $130 \mathrm{kpc}$ which is partly due to the detection limit and partly due to the limit in the survey area. In the MMT survey of HVSs by Brown et al. (2009a), the HVS candidates are selected by a cutoff in the radial velocity, i.e., $v_{\mathrm{rf}} \gtrsim 275 \mathrm{~km} \mathrm{~s}^{-1}$. To account for these selection effects, we adopt the Galactic potential model listed in Section 2 and simulate the radial distribution of HVSs under the assumption of a 


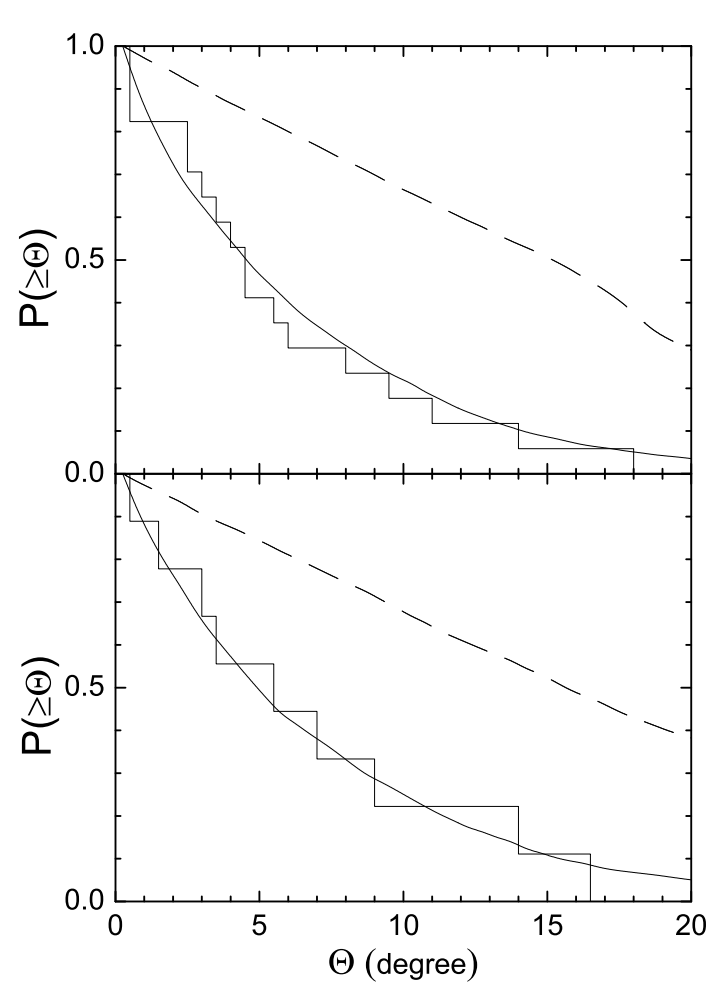

FIG. 11. - Cumulative distributions of HVS inclination angles $(\Theta \mathrm{CDF})$ relative to the two best-fit planes of the detected HVSs. The histograms in the top and bottom panels represent the $\Theta C D F$ of the first and second populations of the detected unbound HVSs, respectively. The solid and dashed curves represent the numerical results from the LP-1 model by adopting the thickness of the two disks which the HVSs are originated from to be $12^{\circ}$ and $13^{\circ}$, respectively (also see Figure 12). The dashed curves show the $\Theta C D F s$ if the stellar binaries are isotropically distributed rather than originated from two disks defined by the two best-fit planes (see details in Section 4.2).

constant HVS ejection rate for each of the above models, which appears to be compatible with current observations (Brown et al. 2007). We only take those HVSs in the surveyed area with the radial distance from $25 \mathrm{kpc}$ to $130 \mathrm{kpc}$ and radial velocities $v_{\mathrm{rf}} \geq 275 \mathrm{~km} \mathrm{~s}^{-1}$ as the simulated sample, which are then used to compare with the observations. The effect of limited lifetime of the ejected HVSs is also considered in our calculations. The selection effects are similarly considered for those models in the $\mathrm{BBH}$ mechanism in Section 5 below. We note here that the $\Theta \mathrm{CDF}$ is not affected much but the $v \mathrm{CDF}$ may be significantly affected by the selection effects.

\section{2. $\Theta \mathrm{CDF}$ : the inclination angle distribution of the ejected stars}

Figure 11 shows the $\Theta C D F$ s obtained from the "RW1 " model. As seen from this figure, the observational $\Theta \mathrm{CDF}$ for both HVS populations can be well reproduced if the HVSs were originated from two disk-like stellar structures with orientations the same as the two best-fit planes and thickness of $\sim 12^{\circ}$ and $13^{\circ}$, respectively (also see the top panel of Figure 12). Here, the thickness of a stellar disk is defined by the standard deviation of the inclination angle of stars in the disk from the disk central plane. For these two populations of the detected HVSs, our K-S tests find the likelihoods of 0.896 and 0.999 that the observational $\Theta \mathrm{CDF}$ are drawn from the same distri-
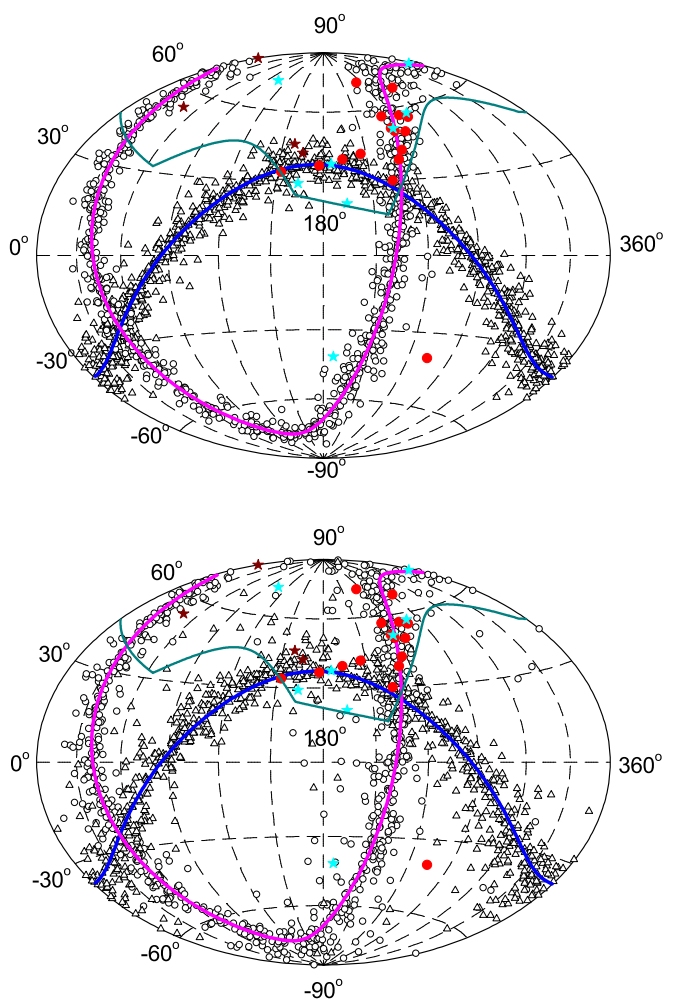

FIG. 12.- Aitoff-projection for both the simulated HVSs and the detected HVSs. The red solid circles, the carmine stars, and the cyan stars represent the detected unbound HVSs, the HVS candidates, and the bound population of HVSs, respectively. The top and bottom panels show the simulated HVSs produced by the TBK mechanism and the BBH mechanism (the LP-1 model), respectively. In the top panel, the open circles and triangles represent the simulated HVSs which are originated from the two best-fit disk planes with normals of $(l, b)=\left(311^{\circ},-14^{\circ}\right)$ and $\left(176^{\circ},-53^{\circ}\right)$, and the thickness of these two originating disks are $12^{\circ}$ and $13^{\circ}$, respectively; while in the bottom panel, the thickness of the two originating disks are $7^{\circ}$ and $10^{\circ}$, respectively. The green solid curve shows the boundary of the survey area in the Northern hemisphere in each panel. This figure shows that the spatial distribution of the detected HVSs can be well reproduced by both the TBK mechanism and the BBH mechanism if the progenitors of the detected HVSs are originated from two thin disks with the same orientations as the fitted ones. One difference in the resulted spatial distributions of these two mechanisms is that a small number of simulated HVSs from the BBH mechanism can have larger inclination angles relative to their original planes, which is due to that some progenitors can interact with the secondary $\mathrm{BH}$ at a very small separation and receive a large kick.

bution as that obtained from the numerical simulations for the "RW-1" model, respectively. All the other models (i.e., "LP-1", "LP-2", "UB-1", "UB-2", and "RW-2") can reproduce the observational $\Theta$ CDFs for both HVS populations by choosing suitable thickness (typically in the range of $7^{\circ}-13^{\circ}$ ) for the two disks. The resulted $\Theta C D F s$ are mainly determined by the thickness of the disks where the HVS progenitors are originated, and also weakly depend on the mechanism adopted that leads to the injection of stellar binaries into the immediate vicinity of the MBH.

Alternatively, assuming that the stellar binaries, i.e., the HVS progenitors, were isotropically distributed but with other initial settings the same as those in the above models, the ejected HVSs should also be isotropically distributed in the survey area. We do similar numerical simulations and obtain the $\Theta \mathrm{CDF}$ for those isotropically 
distributed HVSs, relative to the two best-fit thin disk planes of the detected HVSs, as shown by the dashed curves in Figure 11. Here, a simulated HVS is assigned to one of the two populations if it is closer to the best-fit plane of that population than to the other plane. Using the K-S test, we find the likelihoods of $1 \times 10^{-4}$ and 0.016 that the $\Theta C D F s$ of the simulated HVSs are the same as those from observations for the first and second population of the detect HVSs, respectively. Therefore, we conclude that the detected HVSs are highly unlikely to be produced from the tidal breakup of isotropically distributed progenitorial binary stars. This further strengthens the conclusion obtained in Lu et al. (2010), i.e., the detected HVSs are probably originated from two thin disks with orientations similar to the CWS disk and the northern arm of the minispiral (or the warped outer part of the CWS disk) in the GC, respectively.

\section{3. $v \mathrm{CDF}$ : the velocity distribution of the ejected stars}

Figure 13 shows the $v$ CDFs for both the simulated HVSs (obtained from different models) and the observations. Our numerical simulations show that the $v \mathrm{CDF}$ is almost independent of the thickness of the $\operatorname{disk}(\mathrm{s})$ where the HVS progenitors are originated, but it does depend on how close the stellar binaries can approach the $\mathrm{MBH}$ and on the initial distribution of the semimajor axes of the stellar binaries. Different models produce quite different $v$ CDFs. In the "LP" models and the "UB" models, for example, more than $30 \%$ of the resulted HVSs (with $v_{\mathrm{ej}}^{\infty} \gtrsim 700 \mathrm{~km} \mathrm{~s}^{-1}$ ) have velocities larger than the maximum velocity of the detected HVSs $\left(\sim 1000 \mathrm{~km} \mathrm{~s}^{-1}\right)$, while the "RW" models can produce a steep $v$ CDF which is quite similar to the observational ones. The models with log-normal distributions of $a_{\mathrm{b}, \mathrm{i}}$ produce less HVSs at the high-velocity end because of the fraction of stellar binaries with small $a_{\mathrm{b}, \mathrm{i}}$ (i.e., $\lesssim 0.3 \mathrm{AU}$ ) is substantially smaller compared with that in those models with the Öpik law. For the first HVS population, our K-S tests find $2.7 \times 10^{-6}\left(7.9 \times 10^{-3}\right)$ and $2.5 \times 10^{-4}\left(1.3 \times 10^{-2}\right)$ likelihoods that the $v$ CDFs obtained from the "LP-1" ("UB-1") model and the "LP-2" ("UB-2") model are drawn from the same distribution as the observational ones, respectively, which suggests that the first HVS population is unlikely to be produced by either of the "LP" model and the "UB" model. For the second HVS population, the K-S likelihood is $0.01(0.05)$ and $0.06(0.07)$ for the "LP-1" ("UB-1") model and the "LP-2" ("UB2") model, respectively. These numbers suggest that the second population is not likely to be produced by the "LP" or "UB" models with an initial $a_{\mathrm{b}, \mathrm{i}}$ distribution of $1 / a_{\mathrm{b}, \mathrm{i}}$ but it may not be inconsistent with the "LP" (or "UB") models with a log-normal distribution of $a_{\mathrm{b}, \mathrm{i}}$ (though with limited statistics). However, the $v \mathrm{CDF}$ resulted from the "RW" models appear to be consistent with the observations as the $\mathrm{K}-\mathrm{S}$ tests find the likelihoods of $0.52(0.13)$ and $0.43(0.52)$ that the observational $v$ CDFs of the first (second) HVS population are the same as that obtained from the "RW-1" model and the "RW-2" model, respectively.

Adopting a different form of the Galactic potential may affect the estimation of the $v \mathrm{CDF}$ for the detected HVSs in Section 2 and the selection effects discussed in Section 4.1. For example, if adopting a simple Galac-

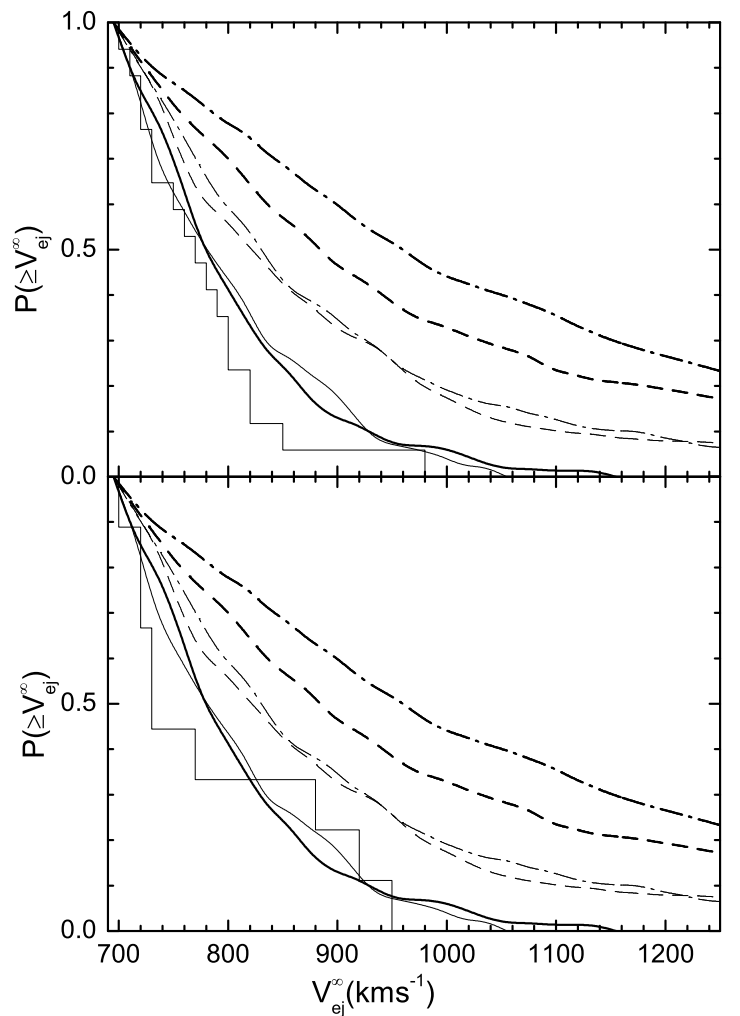

FIG. 13. - Cumulative distribution of the ejection velocity at infinity $(v \mathrm{CDF})$ obtained from different models for the TBK mechanism. The top panel and the bottom panel represent the first and second populations of the detected HVSs, respectively. The thick (thin) dot-dashed, dashed, and solid curves represent the $v \mathrm{CDF}$ obtained from the "LP-1" ("LP-2") model, the "UB-1" ("UB$2 ")$ model, and the "RW-1" ("RW-2") model, respectively. Both the "RW-1" and "RW-2" models can reproduce the observational $v \mathrm{CDF}$, while the other models generate a significant number of HVSs with large $v_{\mathrm{ej}}^{\infty}$ that are not detected by current HVS survey.

tic potential model as that described by Equation (8) in Kenvon et al. (2008), the $v_{\mathrm{ej}}^{\infty}$ of the detected HVSs ranges from $850 \mathrm{~km} \mathrm{~s}^{-1}$ to $1200 \mathrm{~km} \mathrm{~s}^{-1}$ and the slope of the $v \mathrm{CDF}$ is slightly flatter than that estimated in Section 2. The simulated $v$ CDFs from the models of "LP-1", "LP-2", "UB-1", and "UB-2" are not likely to be consistent with the $v \mathrm{CDF}$ of the detected HVSs, while both the "RW-1" model and the "RW-2" model can produce a $v \mathrm{CDF}$ similar to that estimated for the detected HVSs according to the new Galactic potential. Our main conclusion that the TBK mechanism can reproduce the detected $v \mathrm{CDF}$ made in this section is not affected by the choice of the Galactic potential (also see discussion in Sesana et al. (2007) and Kenyon et al. (2008)).

To close this section, we note here that the fraction of stellar binaries resulting in ejection of HVSs with properties similar to the detected ones is around $\sim 3 \%-6 \%$ in those models adopted above (see Table 21). Current observations imply that the total number of HVSs similar to the detected ones is $\sim 100$ (Brown et al. 2007). Therefore, the number of stellar binaries is required to be around a few thousands and the injecting rate is $\sim 10^{-5} \mathrm{yr}^{-1}$. This rate appears to be roughly consistent with that estimated by Madigan et al. (2009) if the progenitors are injected into the immediate vicinity of the $\mathrm{MBH}$ due to secular instability developed in the stellar disk. However, we caution here that the dynamics lead- 


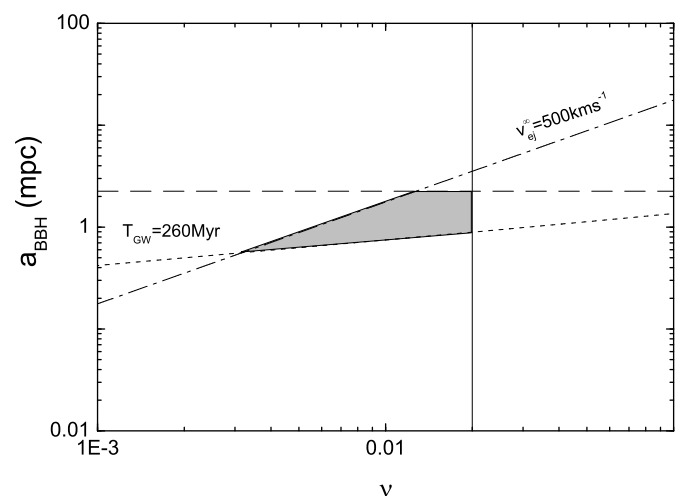

FIG. 14.- Parameter space for the hypothetical $\mathrm{BBH}$ in the $\mathrm{GC}$ if it is responsible for the ejection of the detected HVSs. The short dashed line represents the upper limit of the current semimajor axis of the BBH that is compatible with other observations (Gualandris \& Merritt 2009; Y Yu \& Tremaine 2003). The vertical solid line represents the upper limit of the mass ratio of the $\mathrm{BBH}$ if the secondary BH now exists in the GC. The dot-dashed line is the constraint on the initial semimajor axis of the $\mathrm{BBH}$ from the HVS ejection velocity (see Section 5.1). The dotted line is the constraint on the initial semimajor axis of the BBH from the longest travel time of the detected HVSs, which should be smaller than the decay timescale of the BBH only by gravitational wave radiation. See details in Section 5.1 .

ing to the injection of (binary) stars is currently not clear, and therefore any argument based on the event rate may have substantial limitation in distinguishing the production mechanism of the detected HVSs.

\section{EJECTING HVSS BY A HYPOTHETICAL BBH IN THE $\mathrm{GC}$}

The dynamical interactions between a $\mathrm{BBH}$ and single stars can also eject HVSs (the BBH mechanism). In Section 5.1. we discuss possible constraints on the possible parameter space of a $\mathrm{BBH}$ if it exists (or existed) in the $\mathrm{GC}$ and is responsible for the ejection of the detected HVSs. According to those constraints, in Sections 5.1 5.4 we perform a large number of Monte Carlo simulations for a few models of the decay of the $\mathrm{BBH}$ orbit to investigate whether the spatial and velocity distributions of the detected HVSs are compatible with the $\mathrm{BBH}$ mechanism. ${ }^{5}$

\subsection{Parameter space for the hypothetical BBH}

The parameter space for the secondary $\mathrm{BH}$, if being currently located close to the central primary $\mathrm{MBH}$, has been investigated by Hansen \& Milosavlievic (2003), Yu \& Tremaine (2003), Gillessen et al. (2009), and Gualandris \& Merritt (2009). The observations so far have not shown evidence in contradict with the existence of a $\mathrm{BBH}$ with semimajor axis $a_{\mathrm{BBH}} \lesssim$ $2 \mathrm{mpc}$ and mass ratio $\nu \equiv M_{\bullet .2} / M_{\bullet, 1}<0.02$ (see Figure 2 in $\mathrm{Yu} \&$ Tremaine (2003) and Figure 13 in Gualandris \& Merritt (2009)), where $M_{\bullet, 1}$ and $M_{\bullet, 2}$ are the masses of the two components of the $\mathrm{BBH}$. If a $\mathrm{BBH}$ is responsible for ejecting the detected HVSs, simple constraints on the $\mathrm{BBH}$ parameter space may be directly obtained by the properties of the HVSs as follows (see Figure 14).

5 If multiple intermediate-mass $\mathrm{BHs}$ exist in the GC (Portegies Zwart et al. 2006), HVSs can also be ejected from the $\mathrm{GC}$ by interactions with the BHs; but the stability of the system is not clear and an $N$-body simulation of ejection of HVSs from such a system is beyond the scope of this paper.
- The travel time of the detected HVSs ranges from 30 to 260 Myr so that the $\mathrm{BBH}$ should exist in the GC 260 Myr ago and should not have merged into a single BH 30 Myr ago, which put a stringent constraint on the location of the hypothetical $\mathrm{BBH}$ in the GC. The timescale for the decay of the orbit of a $\mathrm{BBH}$ in the gravitational wave radiation dominated stage is given by (Peters 1964)

$$
\begin{aligned}
& T_{\mathrm{GW}}=\frac{a_{\mathrm{BBH}}}{\left|\dot{a}_{\mathrm{BBH}}\right|} \\
& =\frac{5}{64} \frac{c^{5}}{G^{3}} \frac{a_{\mathrm{BBH}}^{4}}{M_{\bullet, 1} M_{\bullet, 2}\left(M_{\bullet, 1}+M_{\bullet, 2}\right)} f^{-1}(e) \\
& =3.6 \times 10^{7} \mathrm{yr} \frac{f^{-1}(e)}{\nu+\nu^{2}}\left(\frac{a_{\mathrm{BBH}}}{\mathrm{mpc}}\right)^{4}\left(\frac{M_{\bullet, 1}}{4 \times 10^{6} M_{\odot}}\right)^{-3} \text {, }
\end{aligned}
$$

where $\dot{a}_{\mathrm{BBH}}=d a_{\mathrm{BBH}} / d t$ and

$$
f(e) \equiv\left(1-e^{2}\right)^{-7 / 2}\left(1+\frac{73}{24} e^{2}+\frac{37}{96} e^{4}\right) .
$$

Combining Equation (77) and the constraint from the HVS travel time, the hypothetical $\mathrm{BBH}$ should have a semimajor axis $a_{\mathrm{BBH}, 0} \gtrsim$ $2.2\left(\nu+\nu^{2}\right)^{1 / 4} \mathrm{mpc}$ when the detected HVSs began to be ejected out from the GC (see the longdashed line in Figure 14). Note that the above constraint on $a_{\mathrm{BBH}, 0}$ is obtained by assuming that the initial eccentricity of the $\mathrm{BBH}$ is $e_{\mathrm{BBH}, 0}=0$ $\left(f\left(e_{\mathrm{BBH}, 0}\right)=1\right)$ and the $\mathrm{BBH}$ orbital decay is dominated by the energy loss due to gravitational wave radiation (for details see Peters 1964). Adopting a moderately large initial $\mathrm{BBH}$ eccentricity does not change the constraint much (e.g., $a_{\mathrm{BBH}, 0}$ is increased only by a factor of 2.5 if $\left.e_{\mathrm{BBH}, 0}=0.5\right)$. However, the constraint can be changed by orders of magnitude if the initial eccentricity is close to 1 .

- Before the gravitational wave radiation dominated stage, the orbital decay of the $\mathrm{BBH}$ when it is "hard" with $a_{\mathrm{BBH}, 0} \lesssim G M_{\bullet, 2} / 4 \sigma^{2} \simeq$ $430 \nu\left(\sigma / 100 \mathrm{~km} \mathrm{~s}^{-1}\right)^{-2} \mathrm{mpc}($ Quinlan 1996) should be dominated by the interactions of the $\mathrm{BBH}$ with unbound stars in the Galactic bulge or bound stars in the GC stellar cusp (e.g., Yu 2002; Sesana et al. 2008). And the interactions between the BBH and the stars injected from the stellar disk (e.g., the CWS disk) may be insignificant to the $\mathrm{BBH}$ orbital decay as the number of those stars injected into the vicinity of the $\mathrm{BBH}$ is relatively small compared with that from other bound stars. Although HVSs can be ejected out as the high-velocity tail when the $\mathrm{BBH}$ semimajor axis is large (e.g., from $0.2 \mathrm{pc}$ to a few mpc, see Sesana et al. 2008 and Baumgardt et al. 2006), the ejecting efficiency is low and the majority of the ejected high-velocity stars should be old stars from the cusp rather than young stars originated from the stellar disk. If the progenitors of the detected HVSs were originated from the stellar disk surrounding the $\mathrm{MBH}$ and the HVSs are ejected out by the BBH mechanism, the $\mathrm{BBH}$ (with a given mass ratio) should be hard enough to efficiently eject such high-velocity stars. 
The rms velocity of the ejected HVSs at infinity, if their progenitors are on parabolic (or hyperbolic) orbits with eccentricities close to 1 , can be given by (Yu \& Tremaine 2003)

$\sim 740 \mathrm{~km} \mathrm{~s}^{-1}\left(\frac{\nu}{0.01}\right)^{1 / 2}\left(\frac{\mathrm{a}_{\mathrm{BBH}}}{\mathrm{mpc}}\right)^{-1 / 2}\left(\frac{\mathrm{M}_{\bullet}, 1}{4 \times 10^{6} \mathrm{M}_{\odot}}\right)^{1 / 2}$.

Therefore we should have a rough constraint on the $\mathrm{BBH}$ semimajor axis when the $\mathrm{BBH}$ began to eject those detected HVSs, i.e., $a_{\mathrm{BBH}, 0} \lesssim 110 \nu \mathrm{mpc}$, as the minimum velocity of these HVSs at infinity is $\sim 700 \mathrm{~km} \mathrm{~s}^{-1}$ (the solid line in Figure 14). Considering that HVSs can be ejected by the $\mathrm{BBH}$ with a larger semimajor axis, we reset $a_{\mathrm{BBH}, 0} \lesssim 220 \nu \mathrm{mpc}$ which corresponds to an rms ejection velocity of $\sim 500 \mathrm{~km} \mathrm{~s}^{-1}$ and is substantially smaller than the value at which the BBH becomes "hard" Quinlan 1996).

\subsection{Models for the orbital evolution of the $B B H$}

The orbital evolution of a $\mathrm{BBH}$ during its hard stage is dominated by three-body interactions of lowangular momentum stars with the BBH. As shown in Yu \& Tremaine (2003), the decay timescale for the hypothetical $\mathrm{BBH}$ in the $\mathrm{GC}$ is roughly 4-8 Gyr during the hard stage unless the potential of the Galactic bulge is significantly flattened or triaxial (Yu 2002) or there are a large number of massive perturbers (Perets et al. 2007) which lead to efficient transferring of stars onto low-angular momentum orbits. This decay timescale is substantially longer than the travel time of the detected HVSs ( $\leq 260 \mathrm{Myr})$. According to these estimates and the constraints on the parameter space of the $\mathrm{BBH}$, we can use the following three simple models to generally describe the orbital evolution of the $\mathrm{BBH}$ during the course of ejecting the detected HVSs. A few parameters involved in these models are listed in Table 3

(a) If the potential of the Galactic bulge is spherical or at least is not significantly flattened/triaxial and the massive perturbers in the Galactic bulge is not sufficient in quickly filling the loss-cone, then the orbital decay of the $\mathrm{BBH}$ is slow. In this model, we assume that the semimajor axis of the BBH does not change during the period of ejecting the detected HVSs and $a_{\mathrm{BBH}, 0} \simeq 220 \nu \mathrm{mpc}$. (Choosing a somewhat smaller value does not affect our results.)

(b) If the $\mathrm{BBH}$ is initially at $a_{\mathrm{BBH}, 0}=$ $2.2\left(\nu+\nu^{2}\right)^{1 / 4} \mathrm{mpc}$ and the orbital decay is dominated by gravitational wave radiation, we have the evolution of the semimajor axis of an initially circular BBH as $a_{\mathrm{BBH}}=a_{\mathrm{BBH}, 0}(1-t / 260 \mathrm{Myr})^{1 / 4}$. For a $\mathrm{BBH}$ with a moderately large initial eccentricity $e_{\mathrm{BBH}, 0}$, the orbital evolution is similar to the circular one. For a BBH with extremely large initial $e_{\mathrm{BBH}, 0}$ (e.g., see Matsubayashi et al. 2007), the decay timescale is too short and thus the $\mathrm{BBH}$ should not be able to eject HVSs over a time span similar to the longest travel time of the detected HVSs.
TABLE 3

DiFFERENT MODELS FOR THE BBH MECHANISM

\begin{tabular}{|c|c|c|c|c|c|}
\hline$a_{\mathrm{BBH}, 0}$ & $\nu$ & Decay Model & $\theta$ & Origin $^{1}$ & Notations \\
\hline $2.20 \mathrm{mpc}$ & 0.01 & (a) Fixed $a_{\mathrm{BBH}}$ & $\pi / 4$ & Disk & FIX \\
\hline $0.55 \mathrm{mpc}$ & 0.003 & (b) GW dominant & $\pi / 4$ & Disk & GW1 \\
\hline $0.75 \mathrm{mpc}$ & 0.01 & (b) GW dominant & $\pi / 4$ & Disk & GW2 \\
\hline $0.75 \mathrm{mpc}$ & 0.01 & (b) GW dominant & $\pi / 2$ & Disk & GW3 \\
\hline $2.20 \mathrm{mpc}$ & 0.01 & (c) Exponential decay & $\pi / 4$ & Disk & ExpD \\
\hline $2.20 \mathrm{mpc}$ & 0.01 & (a) Fixed $a_{\mathrm{BBH}}$ & $\pi / 4$ & Infinity & FIX-UB \\
\hline
\end{tabular}

1 The origin of the injecting stars is set to be from a disk similar to the CWS disk for the first five models listed here, while it is set to be from infinity and unbound to the BBH for the last model (the "FIX-UB" model).

(c) If the orbital decay of the $\mathrm{BBH}$ during the hard stage is much faster than that of the spherical case because of a highly flattened or triaxial bulge ( $\mathrm{Yu} 2002)$ or many massive perturbers in the bulge (Perets et al. 2007), the semimajor axis of the BBH may quickly evolve from the upper boundary to the lower boundary shown in Figure 14. In order to account for the detected HVSs, this decay timescale should be comparable to the span of the HVS travel time. Otherwise, it is either reduced to model (b) or simply inconsistent with observations. In this model, we assume an exponential evolution for the $\mathrm{BBH}$ semimajor axis, i.e., $a_{\mathrm{BBH}}=a_{\mathrm{BBH}, 0} \exp (-t / 260 \mathrm{Myr}) \simeq$ $220 \nu \exp (-t / 260 \mathrm{Myr}) \mathrm{mpc}$, which corresponds to a constant $a_{\mathrm{BBH}} /\left|\dot{a}_{\mathrm{BBH}}\right|$.

With the above models, we perform Monte-Carlo simulations of the three-body interactions between a single star and the hypothetical $\mathrm{BBH}$ with mass ratios $\nu=0.01$ and 0.003 , respectively (see Table 3 ). For each model, the total number of the three-body experiments is $10^{4}$. We set the tolerance of the fractional energy error to be $10^{-9}$ for each three-body experiment. The initial conditions for the injecting stars are set to be similar to those given in Section 4 for the outer binaries. The initial distribution of the penetration parameters, now defined as $r_{\mathrm{p}} / a_{\mathrm{BBH}, 0}$, is set to be $f\left(r_{\mathrm{p}} / a_{\mathrm{BBH}, 0}\right) \propto$ constant (similar to the "LP" models for the TBK mechanism), and the starting point is $r_{\mathrm{p}, 0} / a_{\mathrm{BBH}, 0} \sim 1.1-1.2$ because the ejection efficiency and velocity drop rapidly for $r_{\mathrm{p}}>r_{\mathrm{p}, 0}$ (e.g., Sesana et al. 2006). The orientation of the BBH relative to the normal of the stellar $\operatorname{disk}(\mathrm{s})$ is set to a few typical values, e.g., $\theta=0, \pi / 4, \pi / 2$, etc., and our calculations show the resulted $\Theta \mathrm{CDF}$ and $v \mathrm{CDF}$ are slightly affected but not much by the choice of this orientation. We can also choose the "RW" models for the BBH mechanism (similar to that for the TBK mechanism), but our calculations show this model do not lead to a suppression of the ejection of HVSs at the high-velocity end.

\section{3. $\Theta \mathrm{CDF}$}

Figure 15 shows the $\Theta$ CDFs obtained from the "GW2" model listed in Table 3 for the BBH mechanism. As seen from the figure, the observational $\Theta C D F$ s can be well reproduced by this model if the HVS progenitors are originated from the two best-fit disks with thickness of $7^{\circ}$ and $10^{\circ}$, respectively (also see the Aitoff-projection of the simulated HVSs in the bottom panel of Figure [12). For these two populations of the detected HVSs, our K-S 


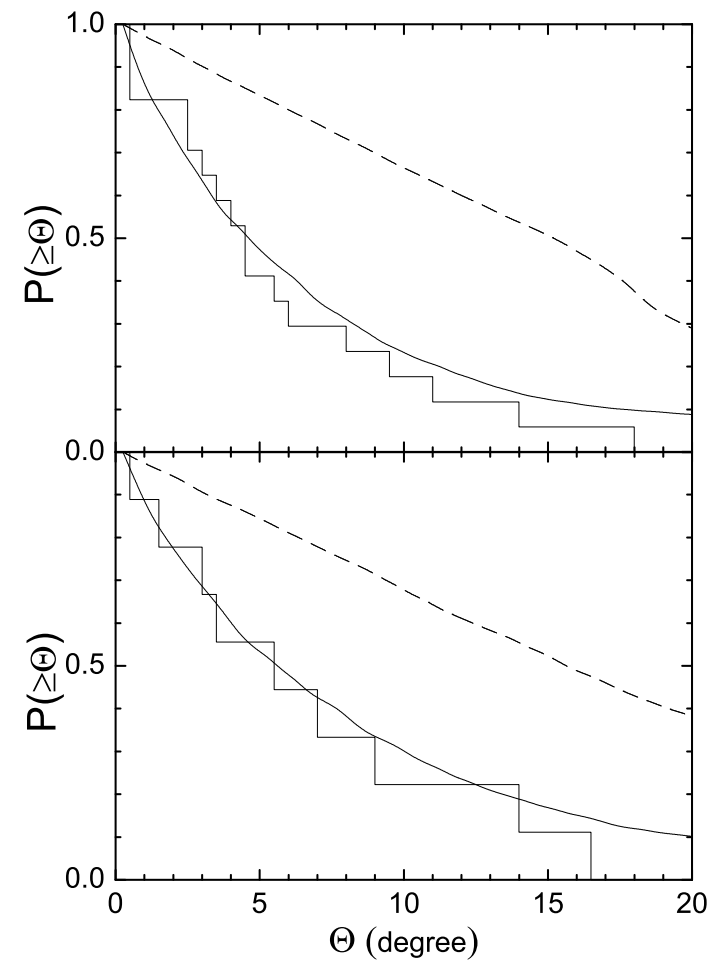

FIG. 15. - Legends are the same as in Figure 10 but the solid curves represent the $\Theta C D F$ obtained from the "GW2" model in the $\mathrm{BBH}$ mechanism. The solid curves in the top and bottom panels show the $\Theta C D F$ of the simulated HVSs which are originated from the two disk-like structures with orientations the same as the two best-fit planes and thickness of $7^{\circ}$ and $10^{\circ}$, respectively.

tests find the likelihoods of 0.97 and 0.91 that the observational $\Theta C D F$ are drawn from the same distributions as that obtained from the numerical simulations for the "GW2" model, respectively. All the other models listed in Table 3 can produce $\Theta$ CDFs similar to the observational ones by choosing suitable thickness of the two disks (typically in the range $7^{\circ}-10^{\circ}$ ) where the HVS progenitors are originated. As seen from Figure 12, a number of HVSs resulted from the BBH mechanism can have substantially large inclination angles relative to their original planes; while no such HVSs appears in the TBK mechanism. The reason for this difference is that there always exist some HVS progenitors which can interact with the secondary $\mathrm{BH}$ at a very small separation and receive a relatively large kick. These very close interactions lead to the large inclination angles of some simulated HVSs. This difference may be helpful in distinguishing the HVS production mechanism in future.

The deviation of the simulated HVSs from the central plane of the disk(s) (with a given thickness) is larger if the mass ratio of the $\mathrm{BBH}$ is larger (see the analysis in Lu et al. 2010). But for the allowed parameter space of the hypothetical BBH as shown in Figure 14, the differences in the resulted $\Theta C D F$ s are quite small for different choices of the mass ratio (and the semimajor axis) and they are easily compensated by slightly different choices of the disk thickness. Similarly, a different choice of the orientation of the disk plane relative to the orbital plane of the BBH may also result in slightly different $\Theta \mathrm{CDF}$, but which can also be compensated by different choices of the thickness of the $\operatorname{disk}(\mathrm{s})$.

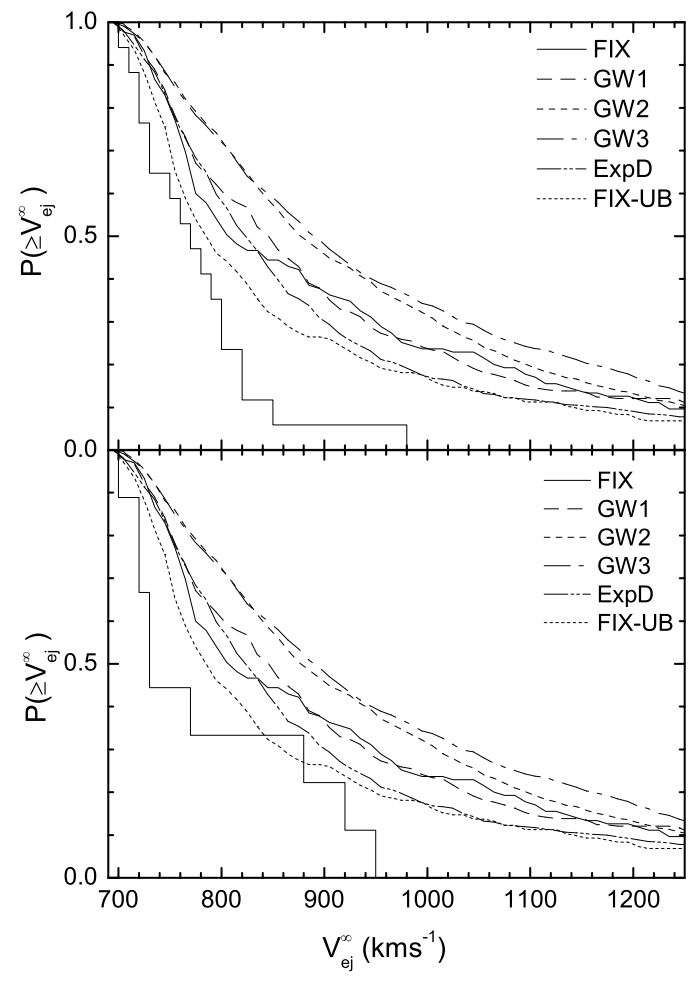

FIG. 16.- Cumulative distribution of the ejection velocity at infinity $(v \mathrm{CDF})$ obtained from different models for the $\mathrm{BBH}$ mechanism. The histograms in the top and bottom panels represent the observational $v$ CDFs for the first and second populations of the detected HVSs, respectively. The solid, long-dashed, short-dotted, dot-dashed, dot-dot-dashed, and dotted curves represent the $v \mathrm{CDF}$ obtained from the "FIX" model, the "GW1" model, the "GW2" model, the "GW3" model, the "ExpD" model, and the "FIX-UB" model, respectively.

\section{4. $v \mathrm{CDF}$}

Figure 16 shows the $v$ CDFs for both the detected HVSs and the simulated HVSs (obtained from different evolutionary models for the BBH mechanism listed in Table 31). Our calculations show that the $v \mathrm{CDF}$ (with $v_{\mathrm{ej}}^{\infty}>700 \mathrm{~km} \mathrm{~s}^{-1}$ ) is almost independent of the choice of the thickness of the disk where the HVS progenitors are originated. The simulated $v \mathrm{CDF}$ is insensitive to the choice of the orientation of the disk relative to the $\mathrm{BBH}$ orbital plane. The simulated $v \mathrm{CDF}$ is also insensitive to the choice of the mass ratio $\nu$ (if $\nu \sim 0.01-0.001$ ) and the eccentricity of the BBH (also see Sesana et al. 2007). As seen from Figure 16, different models produce quite similar $v$ CDFs and too many HVSs with ejection velocities substantially higher than those of the detected HVSs. The primary reason for these similarly flat $v$ CDFs is as follows. The HVS progenitors injected into the region with penetration parameter $r_{\mathrm{p}} / a_{\mathrm{BBH}} \lesssim 1$ can gain some energy during the dynamical interaction with the $\mathrm{BBH}$, and the mean energy gain is mainly determined by the semimajor axis and the mass ratio of the $\mathrm{BBH}$ (Quinlan 1996; Sesana et al. 2006) but insensitive to the detailed values of $r_{\mathrm{p}} / a_{\mathrm{BBH}}$. With increasing $r_{\mathrm{p}} / a_{\mathrm{BBH}}$ to be slightly larger than 1 , the energy gain dramatically drops to 0 (also see Sesana et al. 2006). Therefore, the slope of the $v \mathrm{CDF}$ is largely determined by the scatters of the energy gains around their rms value at $r_{\mathrm{p}} / a_{\mathrm{BBH}} \lesssim 1$, and the scatters are mainly due to the different orbital 
phases of the $\mathrm{BBH}$ and insensitive to other $\mathrm{BBH}$ parameters. Because of these features in the $\mathrm{BBH}$ mechanism, even the "RW" model for injecting stars, which suppresses the ejection of HVSs at the high-velocity end for the TBK mechanism, does not lead to such a suppression for the $\mathrm{BBH}$ mechanism here.

With the relevant parameters for the models listed in Table 3 the simulated $v$ CDFs are all quite flat (except for the "FIX-UB" model), compared with the observational ones. For the first HVS population, our K-S tests find that the likelihoods for the simulated $v \mathrm{CDF}$ to be consistent with the observed distribution are $0.01,0.18$, $0.003,0.0001,0.0001$, and 0.01 for the "FIX", "FIXUB", "GW1", "GW2", "GW3", and "ExpD" models, respectively. Except the high likelihood for the "FIXUB" model, the other small K-S likelihoods above suggest that the first population of the detected HVSs are unlikely drawn from the same distributions as those obtained from the other models. For the second HVS population, the K-S likelihoods are 0.03, 0.12, 0.05, 0.02, 0.02, and 0.04 for the "FIX" , "FIX-UB", "GW1", "GW2", "GW3", and "ExpD" models, respectively. Those likelihoods are not small enough so that the $v \mathrm{CDF}$ for the second HVS population does not appear to be inconsistent with those obtained from the those models, especially for the "FIX-UB" model. For the combined sample of both the first and the second HVS populations, the KS likelihoods are $0.03,0.14,0.003,3 \times 10^{-5}, 3 \times 10^{-5}$, and 0.02 , respectively.

For the models in which $a_{\mathrm{BBH}}$ evolves with time (i.e., the "GW" and "ExpD" models), our simulations indicate a weak dependence of the ejection velocity on time, as the BBH semimajor axis is smaller at a later time and the corresponding ejection velocity is larger (see Equation (9)). Compared to our results, the simulated $v \mathrm{CDF}$ obtained by Sesana et al. (2007) is flatter, as in their model the $\mathrm{BBH}$ decays fast at large separations and more stars with higher velocities are ejected at a later time as the $\mathrm{BBH}$ becomes harder. Due to the limited statistics of the detected HVSs and the uncertainties in the $\mathrm{BBH}$ dynamical evolution model, it appears to be premature to use the $v \mathrm{CDF}$ to distinguish whether the HVSs are ejected due to the TBK mechanism or the BBH mechanism and also constrain detailed dynamical models for injecting stars to the $\mathrm{MBH}(\mathrm{s})$ vicinity.

Note that the ejected stars are actually moving in the Galactic potential, some of those with low velocities (e.g., $v_{\text {ej }}^{\infty} \lesssim 400 \mathrm{~km} \mathrm{~s}^{-1}$ ) may return to the vicinity of the $\mathrm{BBH}$ and receive further kicks, which may change the final $v$ CDF. We check this effect by doing additional threebody experiments for the ejected stars with low ejection velocities. We find that the obtained final $v$ CDFs are only slightly flatter than those shown in Figure 16 and our conclusions above will not be significantly affected.

\section{CONCLUSIONS AND DISCUSSIONS}

In this paper, we first use three-body experiments to study the interactions of the $\mathrm{MBH}$ with binary stars bound to the MBH. We find that the probability of ejecting HVSs is substantially enhanced due to multiple encounters between the $\mathrm{MBH}$ and the stellar binaries injecting into its vicinity even at a distance substantially larger than the tidal breakup radius. Given a penetration parameter (e.g., $D \gtrsim 150$ ), the velocities of the
HVSs ejected after multiple encounters are substantially smaller than those ejected by first encounters because of the excitations of their semimajor axes and eccentricities.

Assuming that the progenitors of the detected HVSs are originated from stellar disk structures, by using Monte Carlo simulations we find the distribution of the inclination of HVSs relative to the disk planes can be well reproduced by both the mechanism of tidal breakup of binary stars and the mechanism of ejecting HVSs by a hypothetical BBH in the GC. However, an isotropical origination of HVS progenitors is inconsistent with the observed inclination distributions. We find that the spatial distribution of HVSs is primarily determined by the geometrical structure(s) that their progenitors originated but less sensitive to whether the ejection is due to tidal breakup of binary stars in the vicinity of the central MBH or dynamical interactions of stars with a BBH. These results strengthen the conclusion in Lu et al. (2010) that the detected HVSs are probably originated from the two disk-like stellar structures in the GC, one of which is probably the CWS disk.

We find that the HVS velocity distribution should encode some dynamical information in the GC environment. Assuming that the detected HVSs were ejected out from the GC by tidal breakup of binary stars, its velocity distribution can be reproduced if the HVS progenitors diffuse onto low angular momentum orbits slowly and most of the progenitorial binaries were broken up at large distances with small ejection velocities. In this scenario, the HVS velocity distribution not only depends on the distribution of the initial semimajor axes of the stellar binaries but also on how the binary stars diffuse onto low angular momentum orbits to reach the vicinity of the central $\mathrm{MBH}$. If the progenitors were injected to the vicinity of the $\mathrm{MBH}$ by large perturbations on their orbital angular momenta, the simulated HVS velocity distributions appear to be flatter at the high-velocity end, which are inconsistent with the observed ones. If the HVSs were ejected out by a BBH in the GC, our simulations show that the HVS velocity distribution is not sensitive to the mass ratio and other properties of the $\mathrm{BBH}$ but may depend on how the BBH orbit decays (cf., Sesana et al. 2007). We find that it is less likely that the observed HVS velocity distribution can be reproduced with the allowed parameter space of the $\mathrm{BBH}$, as the $\mathrm{BBH}$ mechanism produces a relatively flat spectrum at the high-velocity end; however, the BBH mechanism cannot be statistically ruled out, yet. Future deep surveys of HVSs and better statistics of the HVS spatial and velocity distributions should enable to distinguish the ejection mechanisms of HVSs and shed new light on the dynamical environment surrounding the central $\mathrm{MBH}$.

So far, most of the detected HVSs are B-type stars, and their progenitors are associated to some specific stellar structures, such as the CWS disk in the GC. However, there are many late-type stars in the stellar cusp of the GC and injection of these late-type (binary) stars into the vicinity of central $\mathrm{MBH}$ (or $\mathrm{BBH}$ ) should result in an old population of HVSs in the Galactic halo. The spatial distribution of the old population of HVSs should be more isotropic as the parent population of their progenitors is dynamically relaxed. The velocity distribution of this old-population HVSs could also be quite different, as their progenitors may have a different dynami- 
cal environment and are injected into the vicinity of the central MBH differently. Searching for the old population of HVSs (Kollmeier \& Gould 2007; Kollmeier et al. 2009) should be helpful in revealing the dynamical environment in the GC.

Note added in proof: The unbound HVS, HE 04375439 , was proposed to be ejected out from the LMC (Edelmann et al. 2005); and this object was excluded in the original fits to the two disk planes in Lu et al. (2010). However, a recent measurement of its proper motion by the HST suggests it should be originated from the GC (Brown et al. 2010). As seen from Figure 12, the deviation of HE 0437-5439 away from the disk plane(s) is relatively large compared with other HVSs, and it is difficult to have its progenitor originated from the best fit disk(s) under the TBK mechanism. However, the deviation can be compatible with the disk origin under the BBH mechanism in which a small fraction of HVSs with large scatters can be produced (see discussion in Section 5.3). Because of the short lifetime and long traveling time of HE 0437-5439, it is suggested that this object was originally a binary star when being ejected out from the GC and lately merged into a single blue straggler (Brown et al. 2010, Perets 2009b). This proposal is also compatible with the BBH mechanism in which hypervelocity binary stars can be ejected out as predicted in $\mathrm{Lu}$ et al. (2007).

We thank Dr. Warren Brown for helpful conversations on the MMT HVS survey and sending us the data on the boundary of the survey area. This work was supported in part by the BaiRen program from the National Astronomical Observatories, Chinese Academy of Sciences, and the National Natural Science Foundation of China under nos. 10843009, 10973001, and 10973017.

\section{REFERENCES}

Abadi, M. G., Navarro, J. F., \& Steinmetz, M. 2009, ApJ, 691, L63 Antonini, F., Faber, J., Gualandris, A., \& Merritt, D. 2010, ApJ, 713, 90

Bartko, H., et al. 2009, ApJ, 697, 1741

Bartko, H., et al. 2010, ApJ, 708, 834

Baumgardt, H., Gualandris, A., \& Portegies Zwart, S. 2006, MNRAS, 372, 174

Bromley, C. B., Kenyon, S. J., Brown, W. R., \& Geller, M. J. 2009, ApJ, 706, 925

Bromley, C. B., Kenyon, S. J., Geller, M. J., Barcikowski, E., Brown, W. R., \& Kurtz, M. J. 2006, ApJ, 653, 1194

Brown, W. R., Anderson, J., Gnedin, O. Y., Bond, H. E., Geller, M. J., Kenyon, S. J., \& Livio, M. 2010, ApJ, 719, L23

Brown, W. R., Geller, M. J., \& Kenyon, S. J. 2009a, ApJ, 690, 1639

Brown, W. R., Geller, M. J., Kenyon, S. J., \& Bromley, B. C. 2009b, ApJ, 690, L69

Brown, W. R., Geller, M. J., Kenyon, S. J., \& Kurtz, M. J. 2005, ApJ, 622, L33

Brown, W. R., Geller, M. J., Kenyon, S. J., Kurtz, M. J., \& Bromley, B. C. 2007, ApJ, 671, 1708

Do, T., Ghez, A. M., Morris, M. R., Lu, J. R., Matthews, K., Yelda, S., \& Larkin, J. 2009, ApJ, 703, 1323

Dormand, J. R., \& Prince, P. J. 1980, J. Comput. Appl. Math., 6, 19

Duquennoy, A., \& Mayor, M. 1991, A\&A, 248, 485

Edelmann, H., Napiwotzki, R., Heber, U., Christlieb, N. \& Reimers, D. 2005, ApJ, 634, L181

Ghez, A., et al. 2008, ApJ, 689, 1044

Gillessen, S., Eisenhauer, F., Trippe, S., Alexander, T., Genzel, R., Martins, F., \& Ott, T. 2009, ApJ, 692, 1075

Gualandris, A., \& Merritt, D. 2009, ApJ, 705, 361

Gualandris, A., Portegies Zwart, S., \& Sipior, M. S. 2005, MNRAS, 363,223

Hairer, E., Norsett, S. P., \& Wanner, 1993, G. Solving Ordinary Differential Equations. I. Nonstiff Problems, Springer Series in Computational Mathematics, Vol. 8 (Berlin: Springer), 1987

Hansen, B. M. S., 2007, ApJ, 671, L133

Hansen, B. M. S., \& Milosavljevic, M. 2003, ApJ, 593, L77

Heber, U., Edelmann, H., Napiwotzki, R., Altmann, M., \& Scholz, R. D. 2008, A\&A, 483, L21

Hills, J. G. 1988, Nature, 331, 687

Hirsch, H. A., Heber, U., O'Toole, S. J. \& Bresolin, F. 2005, A\&A, 444, L61

Hopman, C. 2009, ApJ, 700, 1933

Kenyon, S. J., Bromley, B. C., Geller, M. J., \& Brown, W. R. 2008, ApJ, 680, 312
Kiminki, D. C., Kobulnicky, H. A., Gilbert, I., Bird, S, \& Chunev, G. 2009, AJ, 137, 4608

Kiminki, D. C., McSwain, M. V., \& Kobulnicky, H. A. 2008, ApJ, 679,1478

Kobulnicky, H. A., \& Fryer, C. L. 2007, ApJ, 670, 747

Kollmeier, J. A., \& Gould, A. 2007, ApJ, 664, 343

Kollmeier, J. A., Gould, A., Knapp, J., \& Beers, T. C. 2009, ApJ, 697,1543

Kroupa, P. 2002, Science, 295, 82

Levin, Y. 2006, ApJ, 653, 1203

Levin, Y., \& Beloborodov, A. 2003, ApJ, 590, L33

Löckmann, U., \& Baumgardt, H. 2008, MNRAS, 384, 323

López-Morales, M., \& Bonanos, A. 2008, ApJ, 685, 47

Lu, J. R., Ghez, A. M., Hornstein, S. D., Morris, M. R., Becklin, E. E., \& Matthews, K. 2009, ApJ, 690, 1463

Lu, Y., Yu, Q., \& Lin, D. N. C. 2007, ApJ, 666, L89

Lu, Y., Zhang, F., \& Yu, Q. 2010, ApJ, 709, 1356

Madigan, A. M., Levin, Y., \& Hopman, C. 2009, ApJ, 697, L44

Matsubayashi, T., Makino, J., \& Ebisuzaki, T. 2007, ApJ, 656, 879

Miyamoto, M., \& Nagai, R. 1975, PASJ, 27, 533

O'Leary, R. M. \& Loeb, A. 2008, MNRAS, 383, 86

Paumard, T., et al. 2006, ApJ, 643, 1011

Perets, H. B. 2009a, ApJ, 690, 795

Perets, H. B. 2009b, ApJ, 698, 1330

Perets, H. B., Hopman, C., \& Alexander, T. 2007, ApJ, 656, 709

Peters, P. C. 1964, Phys. Rev., 136, 1224

Portegies Zwart, S. F., Baumgardt, H., McMillan, S. L. W., Makino, J., Hut, P., \& Ebisuzaki, T. 2006, ApJ, 641, 319

Przybilla, N., Nieva, M. F., Tillich, A., Heber, U., Butler, K., \& Brown, W. R. 2008, A\&A, 488, 51

Quinlan, G. D. 1996, New Astro., 1, 35

Sari, R., Kobayashi, S., \& Ross, E. M. 2010, ApJ, 708, 605

Sesana, A., Haardt, F., \& Madau, P. 2006, ApJ, 651, 392

Sesana, A., Haardt, F., \& Madau, P. 2007, MNRAS, 379, L45

Sesana, A., Haardt, F., \& Madau, P. 2008, ApJ, 686, 432

Torres, G., Andersen, J., \& Giménez, A. 2010, A\&AR, 18, 67

Tutukov, A. V., \& Fedorova, A. V. 2009, Astro. Rep., 53, 839

Wang, B, \& Han, Z. 2009, A\&A, 508, L27

Xue, X. X., et al. 2008, ApJ, 684, 1143

Yu, Q. 2002, MNRAS, 331, 935

Yu, Q., \& Madau, P. 2007, MNRAS, 379, 1293

Yu, Q., \& Tremaine, S. 2003, ApJ, 599, 1129 